\title{
Enhanced production of multi-strange hadrons in high-multiplicity proton-proton collisions
}

\author{
ALICE Collaboration ${ }^{\dagger}$
}

At sufficiently high temperature and energy density, nuclear matter undergoes a transition to a phase in which quarks and gluons are not confined: the quark-gluon plasma (QGP)'. Such an exotic state of strongly interacting quantum chromodynamics matter is produced in the laboratory in heavy nuclei high-energy collisions, where an enhanced production of strange hadrons is observed ${ }^{2-6}$. Strangeness enhancement, originally proposed as a signature of QGP formation in nuclear collisions ${ }^{7}$, is more pronounced for multi-strange baryons. Several effects typical of heavy-ion phenomenology have been observed in high-multiplicity proton-proton (pp) collisions ${ }^{8,9}$, but the enhanced production of multi-strange particles has not been reported so far. Here we present the first observation of strangeness enhancement in high-multiplicity proton-proton collisions. We find that the integrated yields of strange and multi-strange particles, relative to pions, increases significantly with the event charged-particle multiplicity. The measurements are in remarkable agreement with the $\mathrm{p}-\mathrm{Pb}$ collision results ${ }^{10,11}$, indicating that the phenomenon is related to the final system created in the collision. In high-multiplicity events strangeness production reaches values similar to those observed in Pb-Pb collisions, where a QGP is formed.

The production of strange hadrons in high-energy hadronic interactions provides a way to investigate the properties of quantum chromodynamics (QCD), the theory of strongly interacting matter. Unlike up (u) and down (d) quarks, which form ordinary matter, strange (s) quarks are not present as valence quarks in the initial state, yet they are sufficiently light to be abundantly created during the course of the collisions. In the early stages of high-energy collisions, strangeness is produced in hard (perturbative) $2 \rightarrow 2$ partonic scattering processes by flavour creation $(g g \rightarrow s \bar{s}, q \bar{q} \rightarrow s \bar{s})$ and flavour excitation $(g s \rightarrow g s, q s \rightarrow q s)$. Strangeness is also created during the subsequent partonic evolution via gluon splittings $(g \rightarrow s \bar{s})$. These processes tend to dominate the production of high transverse momentum $\left(p_{\mathrm{T}}\right)$ strange hadrons. At low $p_{\mathrm{T}}$, nonperturbative processes dominate the production of strange hadrons. In string fragmentation models the production of strange hadrons is generally suppressed relative to hadrons containing only light quarks, as the strange quark is heavier than up and down quarks. The amount of strangeness suppression in elementary $\left(e^{+} e^{-}\right.$and pp) collisions is an important parameter in Monte Carlo (MC) models. For this reason, measurements of strange hadron production place constraints on these models.

The abundances of strange particles relative to pions in heavyion collisions from top RHIC (Relativistic Heavy-Ion Collider) to LHC (Large Hadron Collider) energies do not show a significant dependence on either the initial volume (collision centrality) or the initial energy density (collision energy). With the exception of the most peripheral collisions, particle ratios are found to be compatible with those of a hadron gas in thermal and chemical equilibrium and can be described using a grand-canonical statistical model $^{12,13}$. In peripheral collisions, where the overlap of the colliding nuclei becomes very small, the relative yields of strange particles to pions decrease and tend toward those observed in pp collisions, for which a statistical-mechanics approach can also be applied ${ }^{14,15}$. Extensions of a pure grand-canonical description of particle production, such as statistical models implementing strangeness canonical suppression ${ }^{16}$ and core-corona superposition ${ }^{17,18}$ models, can effectively produce a suppression of strangeness production in small systems. However, the microscopic origin of enhanced strangeness production is not known, and the measurements presented in this Letter may contribute to its understanding. Several effects, such as azimuthal correlations and mass-dependent hardening of $p_{\mathrm{T}}$ distributions, which in nuclear collisions are typically attributed to the formation of a strongly interacting quark-gluon medium, have been observed in high-multiplicity pp and proton-nucleus collisions at the $\mathrm{LHC}^{8-11,19-25}$. Yet, enhanced production of strange particles as a function of the charged-particle multiplicity density $\left(\mathrm{d} N_{\mathrm{ch}} / \mathrm{d} \eta\right)$ has so far not been observed in pp collisions. The study of pp collisions at high multiplicity is thus of considerable interest as it opens the exciting possibility of a microscopic understanding of phenomena known from nuclear reactions.

In this Letter, we present the multiplicity dependence of the production of primary strange $\left(K_{\mathrm{S}}^{0}, \Lambda, \bar{\Lambda}\right)$ and multi-strange $\left(\Xi^{-}, \bar{\Xi}^{+}, \Omega^{-}, \bar{\Omega}^{+}\right)$hadrons in pp collisions at the centre-of-mass energy of $\sqrt{s}=7 \mathrm{TeV}$. Primary particles are defined as all particles created in the collisions, except those coming from weak decays of light-flavour hadrons and of muons. The measurements have been performed at midrapidity (the particle rapidity is defined as $y=(1 / 2) \ln \left(\left(E+p_{z} c\right) /\left(E-p_{z} c\right)\right)$, where $E$ is the energy and $p_{z}$ is the component of momentum along the beam axis), $|y|<0.5$, with the ALICE detector ${ }^{26}$ at the LHC. Similar measurements of the multiplicity and centrality dependence of strange and multi-strange hadron production have been performed by ALICE in protonlead $(\mathrm{p}-\mathrm{Pb})$ collisions at a centre-of-mass energy per nucleon pair $\sqrt{s_{\mathrm{NN}}}=5.02 \mathrm{TeV}$ (refs 10,11$)$ and in lead-lead $(\mathrm{Pb}-\mathrm{Pb})$ collisions at $\sqrt{s_{\mathrm{NN}}}=2.76 \mathrm{TeV}$ (refs 6,27). The measurements reported here have been obtained in pp collisions at $\sqrt{s}=7 \mathrm{TeV}$ for events having at least one charged particle produced in the pseudorapidity (the particle pseudorapidity is defined as $\eta=-\ln (\tan (\theta / 2))$, where $\theta$ is the angle with respect to the beam axis) interval $|\eta|<1$ (INEL $>0$ ), corresponding to about $75 \%$ of the total inelastic cross-section. To study the multiplicity dependence of strange and multi-strange hadron production, the sample is divided into event classes based on the total ionization energy deposited in the forward detectors, covering the pseudorapidity regions $2.8<\eta<5.1$ and $-3.7<\eta<-1.7$.

Particle/antiparticle production yields are identical within uncertainties. The $p_{\mathrm{T}}$ distributions of $K_{\mathrm{S}}^{0}, \Lambda+\bar{\Lambda}, \Xi^{-}+\bar{\Xi}^{+}$and $\Omega^{-}+\bar{\Omega}^{+}$(in the following denoted as $K_{\mathrm{S}}^{0}, \Lambda, \Xi$ and $\Omega$ ) are shown in Fig. 1 for a selection of event classes with progressively decreasing 


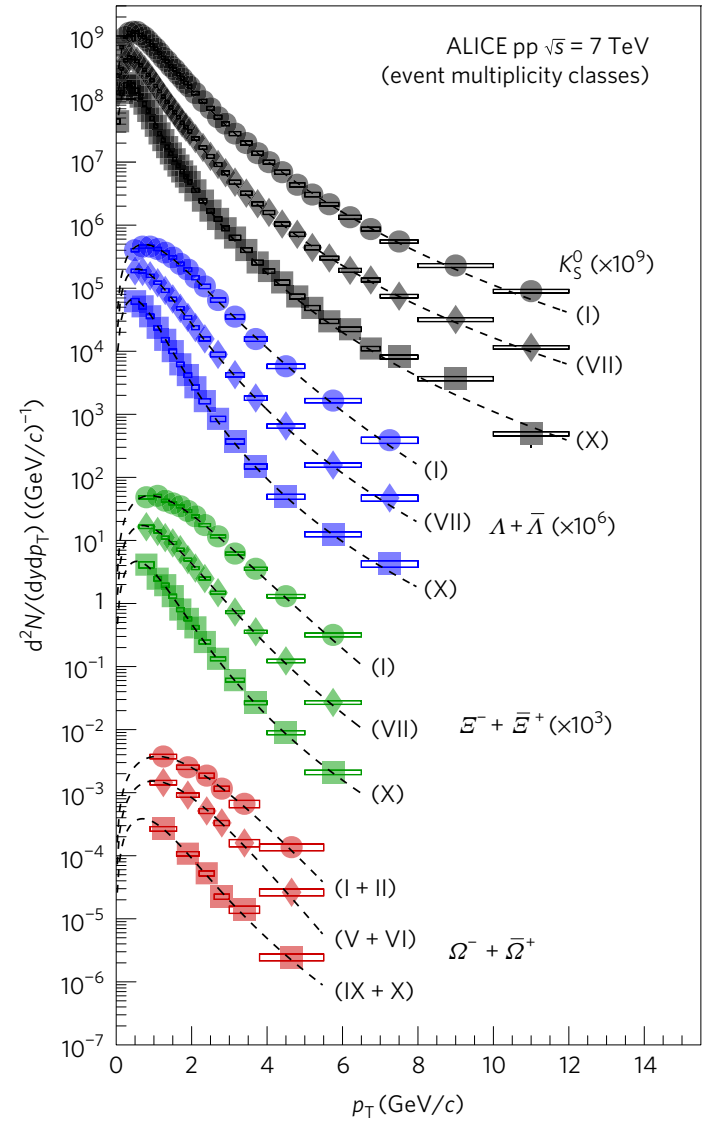

Figure $1 \mid p_{\mathrm{T}}$-differential yields of $K_{\mathrm{S}}^{0}, \Lambda+\bar{\Lambda}, \Xi^{-}+\bar{\Xi}^{+}$and $\Omega^{-}+\bar{\Omega}^{+}$ measured in $|y|<0.5$. The results are shown for a selection of event classes, indicated by roman numbers in brackets, with decreasing multiplicity. The error bars show the statistical uncertainty, whereas the empty boxes show the total systematic uncertainty. The data are scaled by different factors to improve the visibility. The dashed curves represent Tsallis-Lévy fits to each individual distribution to extract integrated yields. The indicated uncertainties all represent standard deviations.

$\left\langle\mathrm{d} N_{\mathrm{ch}} / \mathrm{d} \eta\right\rangle$. The mean pseudorapidity densities of primary charged particles $\left\langle\mathrm{d} N_{\mathrm{ch}} / \mathrm{d} \eta\right\rangle$ are measured at midrapidity, $|\eta|<0.5$. The $p_{\mathrm{T}}$ spectra become harder as the multiplicity increases, with the hardening being more pronounced for higher-mass particles. A similar observation was reported for $\mathrm{p}-\mathrm{Pb}$ collisions $^{10}$, where this and several other features common with $\mathrm{Pb}-\mathrm{Pb}$ collisions are consistent with the appearance of collective behaviour at high multiplicity ${ }^{8,11,19-23}$. In heavy-ion collisions these observations are successfully described by models based on relativistic hydrodynamics. In this framework, the $p_{\mathrm{T}}$ distributions are determined by particle emission from a collectively expanding thermal source ${ }^{28}$. The blast-wave model ${ }^{29}$ is employed to analyse the spectral shapes of $K_{\mathrm{s}}^{0}, \Lambda$ and $\Xi$ in the common highest multiplicity class (class I). A simultaneous fit to all particles is performed following the approach discussed in ref. 10 in the $p_{\mathrm{T}}$ ranges $0-1.5,0.6-2.9$ and $0.6-2.9 \mathrm{GeV} / c$, for $K_{\mathrm{s}}^{0}, \Lambda$ and $\Xi$, respectively. The best fit describes the data to better than $5 \%$ in the respective fit ranges, consistent with particle production from a thermal source at temperature $T_{\mathrm{fo}}$ expanding with a common transverse velocity $\left\langle\beta_{\mathrm{T}}\right\rangle$. The resulting parameters, $T_{\mathrm{fo}_{0}}=163 \pm 10 \mathrm{MeV}$ and $\left\langle\beta_{\mathrm{T}}\right\rangle=0.49 \pm 0.02$, are remarkably similar to the ones obtained in $\mathrm{p}-\mathrm{Pb}$ collisions for an event class with comparable $\left\langle\mathrm{d} N_{\mathrm{ch}} / \mathrm{d} \eta\right\rangle$ (ref. 10).

The $p_{\mathrm{T}}$-integrated yields are computed from the data in the measured ranges and using extrapolations to the unmeasured regions. To extrapolate to the unmeasured region, the data were fitted with a Tsallis-Lévy ${ }^{10}$ parametrization, which gives the best

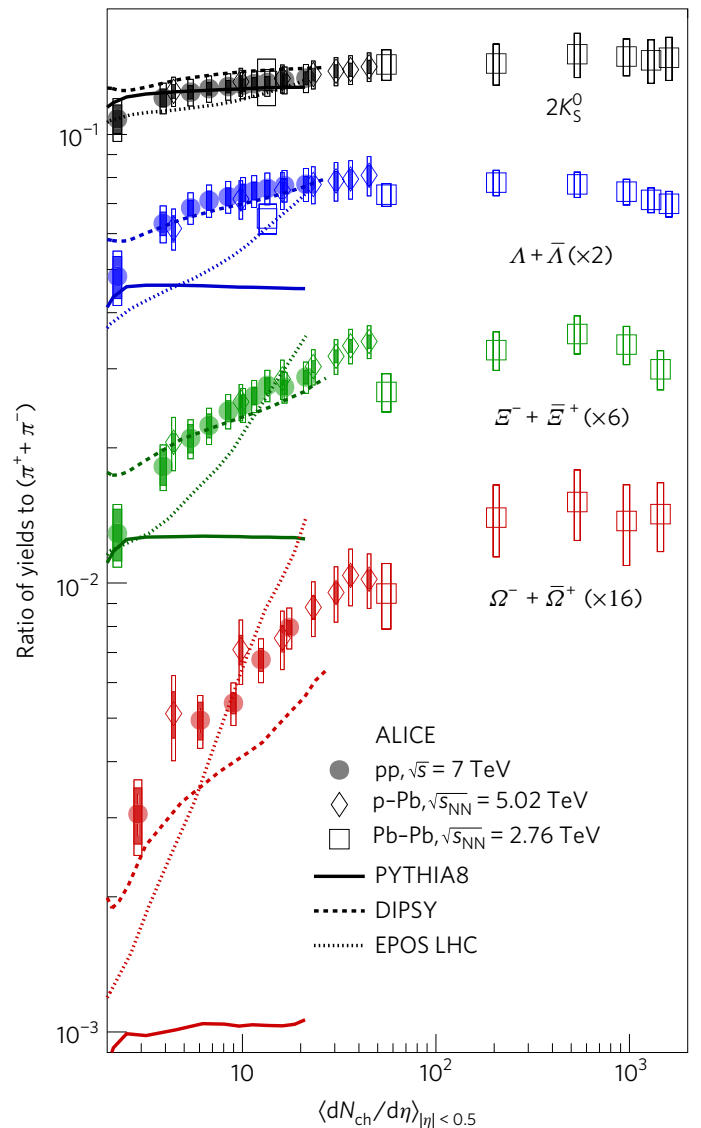

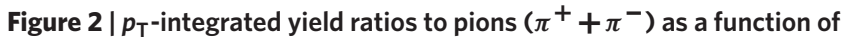
$\left\langle\mathrm{d} N_{\mathrm{ch}} / \mathrm{d} \eta\right\rangle$ measured in $|y|<0.5$. The error bars show the statistical uncertainty, whereas the empty and dark-shaded boxes show the total systematic uncertainty and the contribution uncorrelated across multiplicity bins, respectively. The values are compared to calculations from $\mathrm{MC}$ models $\mathrm{s}^{30-32}$ and to results obtained in $\mathrm{p}-\mathrm{Pb}$ and $\mathrm{Pb}-\mathrm{Pb}$ collisions at the $\mathrm{LHC}^{6,10,11}$. For $\mathrm{Pb}-\mathrm{Pb}$ results the ratio $2 \Lambda /\left(\pi^{+}+\pi^{-}\right)$is shown. The indicated uncertainties all represent standard deviations.

description of the individual spectra for all particles and all event classes over the full $p_{\mathrm{T}}$ range (Fig. 1). Several other fit functions (Boltzmann, $m_{\mathrm{T}}$-exponential, $p_{\mathrm{T}}$-exponential, blast wave, Fermi-Dirac, Bose-Einstein) are employed to estimate the corresponding systematic uncertainties. The fraction of the extrapolated yield for the highest(lowest) multiplicity event class is about $10(25) \%, 16(36) \%, 27(47) \%$ for $\Lambda, \Xi$ and $\Omega$, respectively, and is negligible for $K_{\mathrm{s}}^{0}$. The uncertainty on the extrapolation amounts to about $2(6) \%, 3(10) \%, 4(13) \%$ of the total yield for $\Lambda, \Xi$ and $\Omega$, respectively, and it is negligible for $K_{\mathrm{S}}^{0}$. The total systematic uncertainty on the $p_{\mathrm{T}}$-integrated yields amounts to $5(9) \%, 7(12) \%$, $6(14) \%$ and $9(18) \%$ for $K_{\mathrm{S}}^{0}, \Lambda, \Xi$ and $\Omega$, respectively. A significant fraction of this uncertainty is common to all multiplicity classes and it is estimated to be about $5 \%, 6 \%, 6 \%$ and $9 \%$ for $K_{\mathrm{s}}^{0}, \Lambda, \Xi$ and $\Omega$, respectively. In Fig. 2, the ratios of the yields of $K_{\mathrm{s}}^{0}, \Lambda, \Xi$ and $\Omega$ to the pion $\left(\pi^{+}+\pi^{-}\right)$yield as a function of $\left\langle\mathrm{d} N_{\mathrm{ch}} / \mathrm{d} \eta\right\rangle$ are compared to $\mathrm{p}-\mathrm{Pb}$ and $\mathrm{Pb}-\mathrm{Pb}$ results at the $\mathrm{LHC}^{6,10,11}$. A significant enhancement of strange to non-strange hadron production is observed with increasing particle multiplicity in $\mathrm{pp}$ collisions. The behaviour observed in $\mathrm{pp}$ collisions resembles that of $\mathrm{p}-\mathrm{Pb}$ collisions at a slightly lower centre-of-mass energy ${ }^{11}$, in terms of both the values of the ratios and their evolution with multiplicity. As no significant dependence on the centre-of-mass energy is observed at the LHC for inclusive inelastic collisions, the origin of strangeness production in hadronic collisions is apparently driven by the characteristics of the final state rather than by the collision system or energy. At 


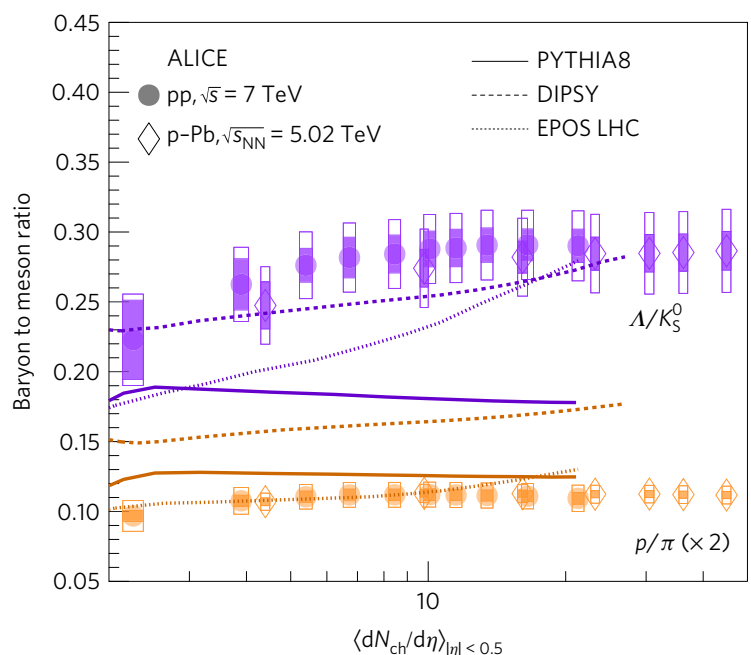

Figure 3 | Particle yield ratios $\Lambda / K_{\mathrm{S}}^{0}=(\Lambda+\bar{\Lambda}) / 2 K_{\mathrm{S}}^{0}$ and $p / \pi=(p+\bar{p}) /\left(\pi^{+}+\pi^{-}\right)$as a function of $\left\langle\mathrm{d} N_{\mathrm{ch}} / \mathrm{d} \eta\right\rangle$. The yield ratios are measured in the rapidity interval $|y|<0.5$. The error bars show the statistical uncertainty, whereas the empty and dark-shaded boxes show the total systematic uncertainty and the contribution uncorrelated across multiplicity bins, respectively. The values are compared to calculations from $\mathrm{MC}$ models $\mathrm{s}^{30-32}$ in pp collisions at $\sqrt{s}=7 \mathrm{TeV}$ and to results obtained in $\mathrm{p}-\mathrm{Pb}$ collisions at the $\mathrm{LHC}^{10}$. The indicated uncertainties all represent standard deviations.

high multiplicity, the yield ratios reach values similar to the ones observed in $\mathrm{Pb}-\mathrm{Pb}$ collisions, where no significant change with multiplicity is observed beyond an initial slight rise. Note that the finalstate average charged-particle density $\left\langle\mathrm{d} N_{\mathrm{ch}} / \mathrm{d} \eta\right\rangle$, which changes by over three orders of magnitude from low-multiplicity pp to central $\mathrm{Pb}-\mathrm{Pb}$, will in general be related to different underlying physics in the various reaction systems. For example, under the assumption that the initial reaction volume in both $\mathrm{pp}$ and $\mathrm{p}-\mathrm{Pb}$ is determined mostly by the size of the proton, $\left\langle\mathrm{d} N_{\mathrm{ch}} / \mathrm{d} \eta\right\rangle$ could be used as a proxy for the initial energy density. In $\mathrm{Pb}-\mathrm{Pb}$ collisions, on the other hand, both the overlap area as well as the energy density could increase with $\left\langle\mathrm{d} N_{\mathrm{ch}} / \mathrm{d} \eta\right\rangle$. Nonetheless, it is a non-trivial observation that particle ratios in $\mathrm{pp}$ and $\mathrm{p}-\mathrm{Pb}$ are identical at the same $\mathrm{d} N_{\mathrm{ch}} / \mathrm{d} \eta$, representing an indication that the final-state particle density might indeed be a good scaling variable between these two systems.

Figure 3 shows that the yield ratios $\Lambda / K_{\mathrm{S}}^{0}=(\Lambda+\bar{\Lambda}) / 2 K_{\mathrm{S}}^{0}$ and $p / \pi=(p+\bar{p}) /\left(\pi^{+}+\pi^{-}\right)$do not change significantly with multiplicity, demonstrating that the observed enhanced production rates of strange hadrons with respect to pions is not due to the difference in the hadron masses. The results in Figs 2 and 3 are compared to calculations from MC models commonly used for pp collisions at the LHC: PYTHIA8 ${ }^{30}$, EPOS LHC ${ }^{31}$ and DIPSY ${ }^{32}$. The kinematic domain and the multiplicity selections are the same for MC and data, namely, dividing the INEL $>0$ sample into event classes based on the total charged-particle multiplicity in the forward region. The observation of a multiplicity-dependent enhancement of the production of strange hadrons along with the constant production of protons relative to pions cannot be simultaneously reproduced by any of the MC models commonly used at the LHC. The model which describes the data best, DIPSY, is a model where interaction between gluonic strings is allowed to form 'colour ropes' which are expected to produce more strange particles and baryons.

To illustrate the evolution of the production of strange hadrons with multiplicity, Fig. 4 presents the yield ratios to pions divided by the values measured in the inclusive INEL $>0 \mathrm{pp}$ sample, both for $\mathrm{pp}$ and $\mathrm{p}-\mathrm{Pb}$ collisions. The observed multiplicity-dependent enhancement with respect to the INEL $>0$ sample follows a hierarchy determined by the hadron strangeness. We have attempted

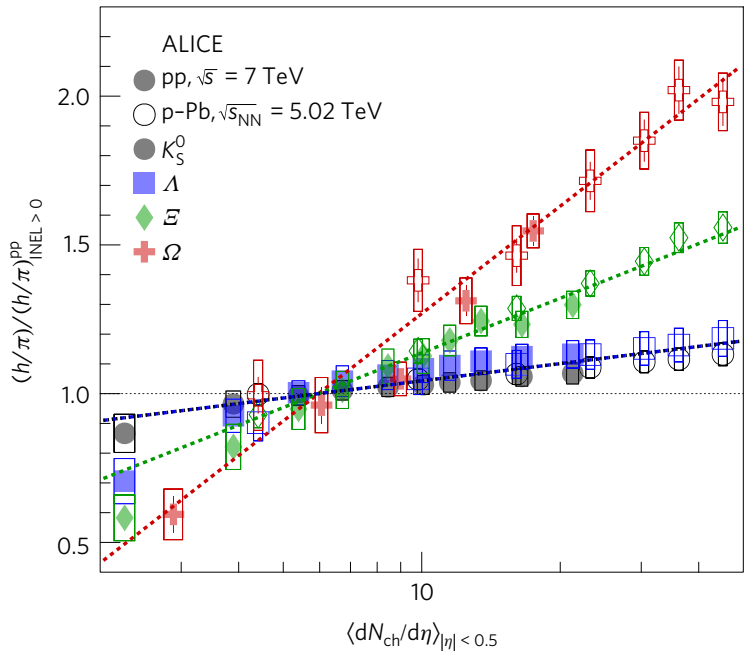

Figure 4 | Particle yield ratios to pions normalized to the values measured in the inclusive INEL $>0 \mathrm{pp}$ sample. The results are shown for $\mathrm{pp}$ and $\mathrm{p}-\mathrm{Pb}$ collisions, both normalized to the inclusive INEL $>0 \mathrm{pp}$ sample. The error bars show the statistical uncertainty. The common systematic uncertainties cancel in the double ratio. The empty boxes represent the remaining uncorrelated uncertainties. The lines represent a simultaneous fit of the results with the empirical scaling formula in equation (1). The indicated uncertainties all represent standard deviations.

to describe the observed strangeness hierarchy by fitting the data presented in Fig. 4 and the empirical function of the form

$$
\frac{(h / \pi)}{(h / \pi)_{\mathrm{INEL}>0}^{\mathrm{pp}}}=1+a S^{b} \log \left[\frac{\left\langle\mathrm{d} N_{\mathrm{ch}} / \mathrm{d} \eta\right\rangle}{\left\langle\mathrm{d} N_{\mathrm{ch}} / \mathrm{d} \eta\right\rangle_{\mathrm{INEL}>0}^{\mathrm{pp}}}\right]
$$

where $S$ is the number of strange or anti-strange valence quarks in the hadron, $(h / \pi)_{\mathrm{INEL}>0}^{\mathrm{pp}}$ and $\left\langle\mathrm{d} N_{\mathrm{ch}} / \mathrm{d} \eta\right\rangle_{\mathrm{INEL}>0}^{\mathrm{pp}}$ are the measured hadron-to-pion ratio and the charged-particle multiplicity density in INEL $>0 \mathrm{pp}$ collisions, respectively, and $a$ and $b$ are free parameters. The fit describes the data well, yielding $a=0.083 \pm$ $0.006, b=1.67 \pm 0.09$, with a $\chi^{2} /$ ndf of 0.66 .

In summary, we have presented the multiplicity dependence of the production of primary strange $\left(K_{\mathrm{s}}^{0}, \Lambda, \bar{\Lambda}\right)$ and multi-strange $\left(\Xi^{-}, \bar{\Xi}^{+}, \Omega^{-}, \bar{\Omega}^{+}\right)$hadrons in pp collisions at $\sqrt{s}=7 \mathrm{TeV}$. The results are obtained as a function of $\left\langle\mathrm{d} N_{\mathrm{ch}} / \mathrm{d} \eta\right\rangle$ measured at midrapidity for event classes selected on the basis of the total charge deposited in the forward region. The $p_{\mathrm{T}}$ spectra become harder as the multiplicity increases. The mass and multiplicity dependences of the spectral shapes are reminiscent of the patterns seen in $\mathrm{p}-\mathrm{Pb}$ and $\mathrm{Pb}-\mathrm{Pb}$ collisions at the LHC, which can be understood assuming a collective expansion of the system in the final state. The data show for the first time in pp collisions that the $p_{\mathrm{T}}$-integrated yields of strange and multi-strange particles relative to pions increase significantly with multiplicity. These particle ratios are similar to those found in $\mathrm{p}-\mathrm{Pb}$ collisions at the same multiplicity densities ${ }^{11}$. The observed enhancement increases with strangeness content rather than with mass or baryon number of the hadron. Such behaviour cannot be reproduced by any of the MC models commonly used, suggesting that further developments are needed to obtain a complete microscopic understanding of strangeness production, and indicating the presence of a phenomenon novel in high-multiplicity pp collisions. The evolution of strangeness enhancement seen at the LHC steadily increases as a function of $\left\langle\mathrm{d} N_{\mathrm{ch}} / \mathrm{d} \eta\right\rangle$ from low-multiplicity $\mathrm{pp}$ to high multiplicity $\mathrm{p}-\mathrm{Pb}$ and reaches the values observed in $\mathrm{Pb}-\mathrm{Pb}$ collisions. This may point towards a common underlying physics mechanism which gradually compensates the strangeness suppression in fragmentation. Further studies extending to higher multiplicity in small systems are essential, as they would 
demonstrate whether strangeness production saturates at the thermal equilibrium values predicted by the grand-canonical statistical model $^{12,13}$ or continues to increase. The remarkable similarity of strange particle production in $\mathrm{pp}, \mathrm{p}-\mathrm{Pb}$ and $\mathrm{Pb}-\mathrm{Pb}$ collisions adds to previous measurements in $\mathrm{pp}$, which also exhibit characteristic features known from high-energy heavy-ion collisions ${ }^{8-11,19-23,25}$ and are understood to be connected to the formation of a deconfined QCD phase at high temperature and energy density.

\section{Methods}

Methods, including statements of data availability and any associated accession codes and references, are available in the online version of this paper.

\section{Received 9 January 2017; accepted 23 March 2017;} published online 24 April 2017

\section{References}

1. Shuryak, E. V. Quantum chromodynamics and the theory of superdense matter. Phys. Rep. 61, 71-158 (1980).

2. Andersen, E. et al. (WA97 Collaboration) Strangeness enhancement at mid-rapidity in $\mathrm{Pb}-\mathrm{Pb}$ collisions at $158 \mathrm{AGeV} / \mathrm{c}$. Phys. Lett. B 449, 401-406 (1999).

3. Afanasiev, S. V. et al. (NA49 Collaboration) $\Xi$ and $\bar{\Xi}$ production in central $\mathrm{Pb}+\mathrm{Pb}$ collisions at $158 \mathrm{GeV} / \mathrm{c}$ per nucleon. Phys. Lett. B 538, 275-281 (2002).

4. Antinori, F. et al. (NA57 Collaboration) Energy dependence of hyperon production in nucleus-nucleus collisions at SPS. Phys. Lett. B 595, 68-74 (2004).

5. Abelev, B. I. et al. (STAR Collaboration) Enhanced strange baryon production in $\mathrm{Au}+\mathrm{Au}$ collisions compared to $\mathrm{p}+\mathrm{p}$ at $\sqrt{s}=200-\mathrm{GeV}$. Phys. Rev. C 77, 044908 (2008).

6. Abelev, B. et al. (ALICE Collaboration) Multi-strange baryon production at mid-rapidity in $\mathrm{Pb}-\mathrm{Pb}$ collisions at $\sqrt{s_{\mathrm{NN}}}=2.76 \mathrm{TeV}$. Phys. Lett. B 728, 216-227 (2014); erratum 734, 409 (2014).

7. Koch, P., Muller, B. \& Rafelski, J. Strangeness in relativistic heavy ion collisions. Phys. Rep. 142, 167-262 (1986).

8. Khachatryan, V. et al. (CMS Collaboration) Observation of long-range near-side angular correlations in proton-proton collisions at the LHC. JHEP $\mathbf{9}$, 091 (2010).

9. Khachatryan, V. et al. (CMS Collaboration) Evidence for collectivity in pp collisions at the LHC. Phys. Lett. B 765, 193-220 (2017).

10. Abelev, B. et al. (ALICE Collaboration) Multiplicity dependence of pion, kaon, proton and lambda production in $\mathrm{p}-\mathrm{Pb}$ collisions at $\sqrt{s_{\mathrm{NN}}}=5.02 \mathrm{TeV}$. Phys. Lett. B 728, 25-38 (2014).

11. Adam, J. et al. (ALICE Collaboration) Multi-strange baryon production in p-Pb collisions at $\sqrt{s_{\mathrm{NN}}}=5.02 \mathrm{TeV}$. Phys. Lett. B 758, 389-401 (2016).

12. Cleymans, J., Kraus, I., Oeschler, H., Redlich, K. \& Wheaton, S. Statistical model predictions for particle ratios at $\sqrt{s_{\mathrm{NN}}}=5.5-\mathrm{TeV}$. Phys. Rev. C $\mathbf{7 4}$ 034903 (2006).

13. Andronic, A., Braun-Munzinger, P. \& Stachel, J. Thermal hadron production in relativistic nuclear collisions: the hadron mass spectrum, the horn, and the QCD phase transition. Phys. Lett. B 673, 142-145 (2009); erratum 678, 516 (2009).

14. Hagedorn, R. \& Ranft, J. Statistical thermodynamics of strong interactions at high-energies. 2. Momentum spectra of particles produced in pp-collisions. Nuovo Cimento Suppl. 6, 169-354 (1968).

15. Becattini, F. \& Heinz, U. W. Thermal hadron production in $\mathrm{p} p$ and $\mathrm{p}$ anti-p collisions. Z. Phys. C 76, 269-286 (1997); erratum 76, 578 (1997).

16. Redlich, K. \& Tounsi, A. Strangeness enhancement and energy dependence in heavy ion collisions. Eur. Phys. J. C 24, 589-594 (2002).

17. Becattini, F. \& Manninen, J. Strangeness production from SPS to LHC. J. Phys. G 35, 104013 (2008).

18. Aichelin, J. \& Werner, K. Centrality dependence of strangeness enhancement in ultrarelativistic heavy ion collisions: a core-corona effect. Phys. Rev. C 79, 064907 (2009); erratum 81, 029902 (2010).

19. Chatrchyan, S. et al. (CMS Collaboration) Observation of long-range near-side angular correlations in proton-lead collisions at the LHC. Phys. Lett. B 718, 795-814 (2013).

20. Abelev, B. et al. (ALICE Collaboration) Long-range angular correlations on the near and away side in $\mathrm{p}-\mathrm{Pb}$ collisions at $\sqrt{s_{\mathrm{NN}}}=5.02 \mathrm{TeV}$. Phys. Lett. B 719, 29-41 (2013)

21. Aad, G. et al. (ATLAS Collaboration) Observation of associated near-side and away-side long-range correlations in $\sqrt{s_{\mathrm{NN}}}=5.02 \mathrm{TeV}$ proton-lead collisions with the ATLAS detector. Phys. Rev. Lett. 110, 182302 (2013).
22. Aad, G. et al. (ATLAS Collaboration) Measurement with the ATLAS detector of multi-particle azimuthal correlations in $\mathrm{p}+\mathrm{Pb}$ collisions at $\sqrt{s_{\mathrm{NN}}}=5.02 \mathrm{TeV}$. Phys. Lett. B 725, 60-78 (2013)

23. Chatrchyan, S. et al. (CMS Collaboration) Multiplicity and transverse momentum dependence of two- and four-particle correlations in $\mathrm{pPb}$ and $\mathrm{PbPb}$ collisions. Phys. Lett. B 724, 213-240 (2013).

24. Abelev, B. B. et al. (ALICE Collaboration) Long-range angular correlations of $\pi, \mathrm{K}$ and $\mathrm{p}$ in $\mathrm{p}-\mathrm{Pb}$ collisions at $\sqrt{s_{\mathrm{NN}}}=5.02 \mathrm{TeV}$. Phys. Lett. B 726, 164-177 (2013).

25. Khachatryan, V. et al. (CMS Collaboration) Multiplicity and rapidity dependence of strange hadron production in $\mathrm{pp}, \mathrm{pPb}$, and $\mathrm{PbPb}$ collisions at the LHC. Phys. Lett. B 768, 103-129 (2017).

26. Aamodt, K. et al. (ALICE Collaboration) The ALICE experiment at the CERN LHC. JINST 3, S08002 (2008).

27. Abelev, B. et al. (ALICE Collaboration) $K_{S}^{0}$ and $\Lambda$ production in $\mathrm{Pb}-\mathrm{Pb}$ collisions at $\sqrt{s_{\mathrm{NN}}}=2.76 \mathrm{TeV}$. Phys. Rev. Lett. 111, 222301 (2013).

28. Heinz, U. W. Concepts of heavy ion physics. In Proc. 2003 CERN-CLAF School of High-Energy Physics (ed. Ellis, N.) 165-238 (CERN, 2004); https://inspirehep.net/record/714564

29. Schnedermann, E., Sollfrank, J. \& Heinz, U. W. Thermal phenomenology of hadrons from $200 \mathrm{~A} / \mathrm{GeV} \mathrm{S}+\mathrm{S}$ collisions. Phys. Rev. C 48, 2462-2475 (1993).

30. Sjöstrand, T., Mrenna, S. \& Skands, P. Z. A brief introduction to PYTHIA 8.1. Comput. Phys. Commun. 178, 852-867 (2008).

31. Pierog, T., Karpenko, I., Katzy, J., Yatsenko, E. \& Werner, K. EPOS LHC: test of collective hadronization with LHC data. Phys. Rev. C 92, 034906 (2015)

32. Bierlich, C. \& Christiansen, J. R. Effects of colour reconnection on hadron flavour observables. Phys. Rev. D 92, 094010 (2015).

\section{Acknowledgements}

The ALICE Collaboration would like to thank all its engineers and technicians for their invaluable contributions to the construction of the experiment and the CERN accelerator teams for the outstanding performance of the LHC complex. The ALICE Collaboration gratefully acknowledges the resources and support provided by all Grid centres and the Worldwide LHC Computing Grid (WLCG) collaboration. The ALICE Collaboration acknowledges the following funding agencies for their support in building and running the ALICE detector: State Committee of Science, World Federation of Scientists (WFS) and Swiss Fonds Kidagan, Armenia; Conselho Nacional de Desenvolvimento Científico e Tecnológico (CNPq), Financiadora de Estudos e Projetos (FINEP), Fundação de Amparo à Pesquisa do Estado de São Paulo (FAPESP); Ministry of Science \& Technology of China (MSTC), National Natural Science Foundation of China (NSFC) and Ministry of Education of China (MOEC)"; Ministry of Science, Education and Sports of Croatia and Unity through Knowledge Fund, Croatia; Ministry of Education and Youth of the Czech Republic; Danish Natural Science Research Council, the Carlsberg Foundation and the Danish National Research Foundation; The European Research Council under the European Community's Seventh Framework Programme; Helsinki Institute of Physics and the Academy of Finland; French CNRS-IN2P3, the 'Region Pays de Loire', 'Region Alsace, 'Region Auvergne' and CEA, France; German Bundesministerium fur Bildung, Wissenschaft, Forschung und Technologie (BMBF) and the Helmholtz Association; General Secretariat for Research and Technology, Ministry of Development, Greece; National Research, Development and Innovation Office (NKFIH), Hungary; Council of Scientific and Industrial Research (CSIR), New Delhi; Department of Atomic Energy and Department of Science and Technology of the Government of India; Istituto Nazionale di Fisica Nucleare (INFN) and Centro Fermi - Museo Storico della Fisica e Centro Studi e Ricerche 'Enrico Fermi', Italy; Japan Society for the Promotion of Science (JSPS) KAKENHI and MEXT, Japan; National Research Foundation of Korea (NRF); Consejo Nacional de Cienca y Tecnologia (CONACYT), Direccion General de Asuntos del Personal Academico(DGAPA), México, Amerique Latine Formation academique - European Commission (ALFA-EC) and the EPLANET Program (European Particle Physics Latin American Network); Stichting voor Fundamenteel Onderzoek der Materie (FOM) and the Nederlandse Organisatie voor Wetenschappelijk Onderzoek (NWO), Netherlands; Research Council of Norway (NFR); Pontificia Universidad Católica del Perú; National Science Centre, Poland; Ministry of National Education/Institute for Atomic Physics and National Council of Scientific Research in Higher Education (CNCSI-UEFISCDI), Romania; Joint Institute for Nuclear Research, Dubna; Ministry of Education and Science of Russian Federation, Russian Academy of Sciences, Russian Federal Agency of Atomic Energy, Russian Federal Agency for Science and Innovations and The Russian Foundation for Basic Research; Ministry of Education of Slovakia; Department of Science and Technology, South Africa; Centro de Investigaciones Energeticas, Medioambientales y Tecnologicas (CIEMAT),

E-Infrastructure shared between Europe and Latin America (EELA), Ministerio de Economía y Competitividad (MINECO) of Spain, Xunta de Galicia (Consellería de Educación), Centro de Aplicaciones Tecnolgicas y Desarrollo Nuclear (CEADEN), Cubaenergía, Cuba, and IAEA (International Atomic Energy Agency); Swedish Research Council (VR) and Knut \& Alice Wallenberg Foundation (KAW); National Science and Technology Development Agency (NSDTA), Suranaree University of Technology (SUT) and Office of the Higher Education Commission under NRU project of Thailand; Ukraine Ministry of Education and Science; United Kingdom Science and Technology Facilities Council (STFC); The United States Department of Energy, the United States National Science Foundation, the State of Texas, and the State of Ohio. 


\section{Author contributions}

All authors have contributed to the publication, being variously involved in the design and the construction of the detectors, in writing software, calibrating subsystems, operating the detectors and acquiring data, and finally analysing the processed data. The ALICE Collaboration members discussed and approved the scientific results. The manuscript was prepared by a subgroup of authors appointed by the collaboration and subject to an internal collaboration-wide review process. All authors reviewed and approved the final version of the manuscript.

\section{Additional information}

Reprints and permissions information is available online at www.nature.com/reprints. Publisher's note: Springer Nature remains neutral with regard to jurisdictional claims in published maps and institutional affiliations. Correspondence and requests for materials should be addressed to ALICE Collaboration.

\section{Competing financial interests}

The authors declare no competing financial interests.

This article is licensed under a Creative Commons Attribution 4.0 International License, which permits use, sharing, adaptation, BY
you give appropriate credit to the original author(s) and the source, provide a link to the Creative Commons license, and indicate if changes were made.

The images or other third party material in this article are included in the article's Creative Commons license, unless indicated otherwise in a credit line to the material. If material is not included in the article's Creative Commons license and your intended use is not permitted by statutory regulation or exceeds the permitted use, you will need to obtain permission directly from the copyright holder. To view a copy of this license, visit http://creativecommons.org/licenses/by/4.0/. 


\section{ALICE Collaboration}

J. Adam ${ }^{40}$, D. Adamová ${ }^{86}$, M. M. Aggarwal ${ }^{90}$, G. Aglieri Rinella ${ }^{36}$, M. Agnello $^{32,112}$, N. Agrawal ${ }^{49}$, Z. Ahammed $^{135}$, S. Ahmad ${ }^{19}$, S. U. Ahn ${ }^{70}$, S. Aiola ${ }^{139}$, A. Akindinov ${ }^{60}$, S. N. Alam ${ }^{135}$, D. S. D. Albuquerque ${ }^{123}$, D. Aleksandrov ${ }^{82}$, B. Alessandro ${ }^{112}$, D. Alexandre ${ }^{103}$, R. Alfaro Molina ${ }^{66}$, A. Alici ${ }^{12,106}$, A. Alkin' ${ }^{3}$ J. Alme ${ }^{18,38}$, T. Alt ${ }^{43}$, S. Altinpinar ${ }^{18}$, I. Altsybeev ${ }^{134}$, C. Alves Garcia Prado ${ }^{122}$, M. An ${ }^{7}$, C. Andrei ${ }^{80}$, H. A. Andrews ${ }^{103}$, A. Andronic ${ }^{99}$, V. Anguelov ${ }^{96}$, T. Antičić ${ }^{100}$, F. Antinori ${ }^{109}$, P. Antonioli106, L. Aphecetche ${ }^{115}$, H. Appelshäuser ${ }^{55}$, S. Arcelli 27, R. Arnaldi172, O. W. Arnold ${ }^{37,95}$, I. C. Arsene ${ }^{22}$, M. Arslandok ${ }^{55}$, B. Audurier ${ }^{115}$, A. Augustinus ${ }^{36}$, R. Averbeck ${ }^{99}$, M. D. Azmi ${ }^{19}$, A. Badalà108, Y. W. Baek ${ }^{69}$, S. Bagnasco ${ }^{112}$, R. Bailhache ${ }^{55}$, R. Bala ${ }^{93}$, S. Balasubramanian ${ }^{139}$, A. Baldisseri ${ }^{15}$, R. C. Baral ${ }^{63}$, A. M. Barbano ${ }^{26}$, R. Barbera ${ }^{28}$, F. Barile ${ }^{33}$, G. G. Barnaföldi ${ }^{138}$,

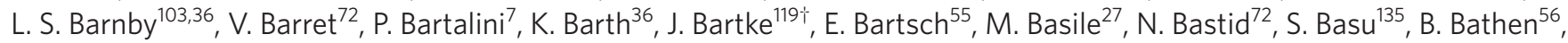
G. Batigne ${ }^{115}$, A. Batista Camejo ${ }^{72}$, B. Batyunya ${ }^{68}$, P. C. Batzing 22, I. G. Bearden ${ }^{83}$, H. Beck ${ }^{55,96}$, C. Bedda ${ }^{112}$, N. K. Behera ${ }^{52}$, I. Belikov ${ }^{57}$, F. Bellini2 ${ }^{27}$, H. Bello Martinez ${ }^{2}$, R. Bellwied ${ }^{124}$, R. Belmont ${ }^{137}$, E. Belmont-Moreno ${ }^{66}$, L. G. E. Beltran ${ }^{121}$, V. Belyaev ${ }^{77}$, G. Bencedi ${ }^{138}$, S. Beole ${ }^{26}$, I. Berceanu ${ }^{80}$, A. Bercuci ${ }^{80}$, Y. Berdnikov ${ }^{88}$, D. Berenyi ${ }^{138}$, R. A. Bertens ${ }^{59}$, D. Berzano ${ }^{36}$, L. Betev $^{36}$, A. Bhasin ${ }^{93}$, I. R. Bhat ${ }^{93}$, A. K. Bhati ${ }^{90}$, B. Bhattacharjee ${ }^{45}$, J. Bhom ${ }^{119}$, L. Bianchi ${ }^{124}$, N. Bianchi ${ }^{74}$, C. Bianchin ${ }^{137}$, J. Bielčík ${ }^{40}$, J. Bielčíková86, A. Bilandzic ${ }^{83,37,95}$, G. Biro ${ }^{138}$, R. Biswas ${ }^{4}$, S. Biswas ${ }^{4,81}$, S. Bjelogrlic ${ }^{59}$, J. T. Blair ${ }^{120}$, D. Blau ${ }^{82}$, C. Blume ${ }^{55}$, F. Bock ${ }^{76,96}$, A. Bogdanov77, H. Bøggild ${ }^{83}$, L. Boldizsár ${ }^{138}$, M. Bombaraa ${ }^{41}$, M. Bonora ${ }^{36}$, J. Book ${ }^{55}$, H. Borel ${ }^{15}$, A. Borissov ${ }^{98}$, M. Borri ${ }^{126,85}$, F. Bossú ${ }^{67}$, E. Botta ${ }^{26}$, C. Bourjau ${ }^{83}$, P. Braun-Munzinger ${ }^{99}$, M. Bregant ${ }^{122}$, T. Breitner ${ }^{54}$, T. A. Broker ${ }^{55}$, T. A. Browning 97, M. Broz ${ }^{40}$, E. J. Brucken ${ }^{47}$, E. Bruna ${ }^{112}$, G. E. Bruno ${ }^{33}$, D. Budnikov ${ }^{101}$, H. Buesching ${ }^{55}$, S. Bufalino ${ }^{32,36}$, P. Buncic ${ }^{36}$, O. Busch ${ }^{130}$, Z. Buthelezi ${ }^{67}$, J. B. Butt ${ }^{16}$, J. T. Buxton ${ }^{20}$, J. Cabala17, D. Caffarri ${ }^{36}$, X. Cai ${ }^{7}$, H. Caines ${ }^{139}$, L. Calero Diaz ${ }^{74}$, A. Caliva ${ }^{59}$, E. Calvo Villar ${ }^{104}$, P. Camerini ${ }^{25}$, F. Carena ${ }^{36}$, W. Carena ${ }^{36}$, F. Carnesecchi ${ }^{27}$, J. Castillo Castellanos ${ }^{15}$, A. J. Castro ${ }^{127}$, E. A. R. Casula ${ }^{24}$, C. Ceballos Sanchez ${ }^{9}$, J. Cepila ${ }^{40}$, P. Cerello ${ }^{112}$, J. Cerkala117, B. Chang ${ }^{125}$, S. Chapeland ${ }^{36}$, M. Chartier ${ }^{126}$, J. L. Charvet ${ }^{15}$, S. Chattopadhyay ${ }^{135}$, S. Chattopadhyay ${ }^{102}$, A. Chauvin ${ }^{95,37}$, V. Chelnokov ${ }^{3}$, M. Cherney ${ }^{89}$, C. Cheshkov ${ }^{132}$, B. Cheynis ${ }^{132}$, V. Chibante Barroso ${ }^{36}$, D. D. Chinellato ${ }^{123}$, S. Cho ${ }^{52}$, P. Chochula ${ }^{36}$, K. Choi ${ }^{98}$, M. Chojnacki ${ }^{83}$, S. Choudhury' ${ }^{135}$, P. Christakoglou ${ }^{84}$, C. H. Christensen ${ }^{83}$, P. Christiansen ${ }^{34}$, T. Chujo ${ }^{130}$, S. U. Chung ${ }^{98}$, C. Cicalo ${ }^{107}$, L. Cifarelli ${ }^{12,27}$, F. Cindolo ${ }^{106}$, J. Cleymans ${ }^{92}$, F. Colamaria ${ }^{33}$, D. Colella ${ }^{61,36}$, A. Collu ${ }^{76}$, M. Colocci $^{27}$ G. Conesa Balbastre ${ }^{73}$, Z. Conesa del Valle ${ }^{53}$, M. E. Connors ${ }^{139}$, J. G. Contreras ${ }^{40}$, T. M. Cormier ${ }^{87}$, Y. Corrales Morales ${ }^{26,112}$, I. Cortés Maldonado ${ }^{2}$, P. Cortese ${ }^{31}$, M. R. Cosentino ${ }^{122}$, F. Costa ${ }^{36}$, J. Crkovska ${ }^{53}$, P. Crochet ${ }^{72}$, R. Cruz Albino ${ }^{11}$, E. Cuautle ${ }^{65}$, L. Cunqueiro ${ }^{56,36}$, T. Dahms ${ }^{95,37}$, A. Dainese ${ }^{109}$, M. C. Danisch ${ }^{96}$, A. Danu ${ }^{64}$, D. Das ${ }^{102}$, I. Das ${ }^{102}$, S. Das ${ }^{4}$, A. Dash ${ }^{81}$, S. Dash ${ }^{49}$, S. De ${ }^{122}$, A. De Caro ${ }^{12,30}$, G. de Cataldo ${ }^{105}$, C. de Conti ${ }^{122}$, J. de Cuveland ${ }^{43}$, A. De Falco ${ }^{24}$, D. De Gruttola ${ }^{12,30}$, N. De Marco ${ }^{112}$, S. De Pasquale ${ }^{30}$, R. D. De Souza ${ }^{123}$, A. Deisting 96,99 , A. Deloff ${ }^{79}$, E. Dénes ${ }^{138 \dagger}$, C. Deplano ${ }^{84}$, P. Dhankher ${ }^{49}$, D. Di Bari3 ${ }^{33}$, A. Di Mauro ${ }^{36}$, P. Di Nezza ${ }^{74}$, B. Di Ruzza ${ }^{109}$, M. A. Diaz Corchero ${ }^{10}$, T. Dietele ${ }^{92}$, P. Dillenseger ${ }^{55}$, R. Divià ${ }^{36}, \varnothing$. Djuvsland ${ }^{18}$, A. Dobrin ${ }^{84,64}$, D. Domenicis Gimenez ${ }^{122}$, B. Dönigus ${ }^{55}$, O. Dordic ${ }^{22}$, T. Drozhzhova ${ }^{55}$, A. K. Dubey ${ }^{135}$, A. Dubla ${ }^{59}$, L. Ducroux ${ }^{132}$, P. Dupieux ${ }^{72}$, R. J. Ehlers ${ }^{139}$, D. Elia ${ }^{105}$, E. Endress ${ }^{104}$, H. Engel ${ }^{54}$, E. Epple ${ }^{139}$, B. Erazmus ${ }^{115}$, I. Erdemir ${ }^{55}$, F. Erhardt Eri1, $^{131}$, B. Espagnon ${ }^{53}$, M. Estienne ${ }^{115}$, S. Esumi ${ }^{130}$, J. Eum ${ }^{98}$, D. Evans ${ }^{103}$, S. Evdokimov ${ }^{113}$, G. Eyyubova ${ }^{40}$, L. Fabbietti ${ }^{95,37}$, D. Fabris ${ }^{109}$, J. Faivre ${ }^{73}$, A. Fantoni ${ }^{74}$, M. Fasel ${ }^{76}$, L. Feldkamp ${ }^{56}$, A. Feliciello ${ }^{112}$, G. Feofilov ${ }^{134}$, J. Ferencei ${ }^{86}$, A. Fernández Téllez ${ }^{2}$, E. G. Ferreiro ${ }^{17}$, A. Ferretti ${ }^{26}$, A. Festanti2 ${ }^{29}$, V. J. G. Feuillard ${ }^{15,72}$, J. Figiel ${ }^{119}$, M. A. S. Figueredo ${ }^{126,122}$, S. Filchagin ${ }^{101}$, D. Finogeev ${ }^{58}$, F. M. Fionda ${ }^{24}$, E. M. Fiore ${ }^{33}$, M. Floris ${ }^{36}$, S. Foertsch ${ }^{67}$, P. Foka ${ }^{99}$, S. Fokin ${ }^{82}$, E. Fragiacomo ${ }^{111}$, A. Francescon ${ }^{36}$, A. Francisco ${ }^{115}$, U. Frankenfeld ${ }^{99}$, G. G. Fronze ${ }^{26}$, U. Fuchs ${ }^{36}$, C. Furget ${ }^{73}$, A. Furs ${ }^{58}$, M. Fusco Girard ${ }^{30}$, J. J. Gaardhøje ${ }^{83}$, M. Gagliardi²6, A. M. Gago ${ }^{104}$, K. Gajdosova ${ }^{83}$, M. Gallio ${ }^{26}$, C. D. Galvan ${ }^{121}$, D. R. Gangadharan ${ }^{76}$, P. Ganoti91, C. Gao ${ }^{7}$, C. Garabatos $^{99}$, E. Garcia-Solis ${ }^{13}$, K. Garg 28 , C. Gargiulo36, P. Gasik ${ }^{95,37}$, E. F. Gauger ${ }^{120}$, M. Germain ${ }^{115}$, M. Gheata ${ }^{36,64}$, P. Ghosh ${ }^{135}$, S. K. Ghosh ${ }^{4}$, P. Gianotti ${ }^{74}$, P. Giubellino ${ }^{112,36}$, P. Giubilato ${ }^{29}$, E. Gladysz-Dziadus ${ }^{19}$, P. Glässel ${ }^{96}$, D. M. Goméz Coral ${ }^{66}$, A. Gomez Ramirez ${ }^{54}$, A. S. Gonzalez ${ }^{36}$, V. Gonzalez ${ }^{10}$, P. González-Zamora ${ }^{10}$, S. Gorbunov³, L. Görlich ${ }^{119}$, S. Gotovac ${ }^{118}$, V. Grabski ${ }^{66}$, O. A. Grachov' ${ }^{139}$, L. K. Graczykowski ${ }^{136}$, K. L. Graham ${ }^{103}$, A. Grelli59, A. Grigoras ${ }^{36}$, C. Grigoras ${ }^{36}$, V. Grigoriev7, A. Grigoryan', S. Grigoryan ${ }^{68}$, B. Grinyov ${ }^{3}$, N. Grion ${ }^{111}$, J. M. Gronefeld ${ }^{99}$, J. F. Grosse-Oetringhaus ${ }^{36}$, R. Grosso 99 , L. Gruber ${ }^{114}$, F. Guber ${ }^{58}$, R. Guernane ${ }^{73}$, B. Guerzoni ${ }^{27}$, K. Gulbrandsen ${ }^{83}$, T. Gunji129, A. Gupta ${ }^{93}$, R. Gupta93, R. Haake ${ }^{56,36}$, C. Hadjidakis ${ }^{53}$, M. Haiduc ${ }^{64}$, H. Hamagaki ${ }^{129}$, G. Hamar ${ }^{138}$, J. C. Hamon ${ }^{57}$, J. W. Harris ${ }^{139}$, A. Harton ${ }^{13}$, D. Hatzifotiadou ${ }^{106}$, S. Hayashi' ${ }^{29}$, S. T. Heckel ${ }^{55}$, E. Hellbär ${ }^{55}$, H. Helstrup ${ }^{38}$, A. Herghelegiu ${ }^{80}$, G. Herrera Corral ${ }^{11}$, F. Herrmann ${ }^{56}$, B. A. Hess ${ }^{35}$, K. F. Hetland ${ }^{38}$, H. Hillemanns ${ }^{36}$, B. Hippolyte ${ }^{57}$, D. Horak ${ }^{40}$, R. Hosokawa ${ }^{130}$, P. Hristov ${ }^{36}$, C. Hughes ${ }^{127}$, T. J. Humanic ${ }^{20}$, N. Hussain ${ }^{45}$, T. Hussain ${ }^{19}$, D. Hutter ${ }^{43}$, D. S. Hwang ${ }^{21}$, R. Ilkaev¹01, M. Inaba ${ }^{130}$, E. Incani ${ }^{24}$, M. Ippolitov7,82, M. Irfan ${ }^{19}$, V. Isakov ${ }^{58}$, M. Ivanov 99,36, V. Ivanov ${ }^{88}$, V. Izucheev ${ }^{113}$, B. Jacak ${ }^{76}$, N. Jacazio ${ }^{27}$, P. M. Jacobs ${ }^{76}$, M. B. Jadhav ${ }^{49}$, S. Jadlovska917, J. Jadlovsky ${ }^{117,61}$, C. Jahnke ${ }^{122}$, M. J. Jakubowska ${ }^{136}$, M. A. Janik ${ }^{136}$, P. H. S. Y. Jayarathna ${ }^{124}$, C. Jena ${ }^{29}$, S. Jena ${ }^{124}$, R. T. Jimenez Bustamante ${ }^{99}$, P. G. Jones ${ }^{103}$, A. Jusko ${ }^{103}$, P. Kalinak ${ }^{61}$, A. Kalweit ${ }^{36}$, J. H. Kang ${ }^{140}$, V. Kaplin ${ }^{77}$, S. Kar ${ }^{135}$, A. Karasu Uysal ${ }^{71}$, O. Karavichev ${ }^{58}$, T. Karavicheva ${ }^{58}$, L. Karayan ${ }^{96,99}$, E. Karpechev ${ }^{58}$, U. Kebschull ${ }^{54}$, R. Keidel141 , D. L. D. Keijdener ${ }^{59}$, M. Keil ${ }^{36}$, M. Mohisin Khan ${ }^{19}$, P. Khan ${ }^{102}$, S. A. Khan ${ }^{135}$, A. Khanzadeev ${ }^{88}$, Y. Kharlov ${ }^{113}$, A. Khatun ${ }^{19}$, B. Kileng ${ }^{38}$, D. W. Kim ${ }^{44}$, D. J. Kim²5, D. Kim ${ }^{140}$, H. Kim4,

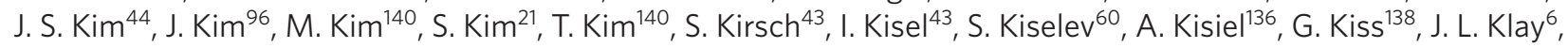
C. Klein ${ }^{55}$, J. Klein ${ }^{36}$, C. Klein-Bösing ${ }^{56}$, S. Klewin ${ }^{96}$, A. Kluge ${ }^{36}$, M. L. Knichel ${ }^{96}$, A. G. Knospe ${ }^{120,124}$, C. Kobdaj ${ }^{16}$, M. Kofarago ${ }^{36}$, T. Kollegger ${ }^{99}$, A. Kolojvari ${ }^{134}$, V. Kondratiev ${ }^{134}$, N. Kondratyeva ${ }^{77}$, E. Kondratyuk ${ }^{113}$, A. Konevskikh ${ }^{58}$, M. Kopcik ${ }^{117}$, M. Kour ${ }^{93}$, C. Kouzinopoulos ${ }^{36}$, O. Kovalenko ${ }^{79}$, V. Kovalenko ${ }^{134}$, M. Kowalski ${ }^{119}$, G. Koyithatta Meethaleveedu ${ }^{49}$, I. Králik ${ }^{61}$, 
A. Kravčáková41 , M. Krivda ${ }^{61,03}$, F. Krizek ${ }^{86}$, E. Kryshen ${ }^{88,36}$, M. Krzewicki ${ }^{43}$, A. M. Kubera ${ }^{20}$, V. Kučera ${ }^{86}$, C. Kuhn ${ }^{57}$, P. G. Kuijer ${ }^{84}$, A. Kumar ${ }^{93}$, J. Kumar ${ }^{49}$, L. Kumar ${ }^{90}$, S. Kumar ${ }^{49}$, P. Kurashvili ${ }^{79}$, A. Kurepin ${ }^{58}$, A. B. Kurepin ${ }^{58}$, A. Kuryakin ${ }^{101}$, M. J. Kweon ${ }^{52}$, Y. Kwon ${ }^{140}$, S. L. La Pointe ${ }^{43,112}$, P. La Rocca ${ }^{28}$, P. Ladron de Guevara11, C. Lagana Fernandes ${ }^{122}$, I. Lakomov ${ }^{36}$, R. Langoy ${ }^{42}$, K. Lapidus ${ }^{37,139}$, C. Lara ${ }^{54}$, A. Lardeux ${ }^{15}$, A. Lattuca ${ }^{26}$, E. Laudi ${ }^{36}$, R. Lea ${ }^{25}$, L. Leardini ${ }^{96}$, S. Lee ${ }^{140}$, F. Lehas ${ }^{84}$, S. Lehner ${ }^{114}$, R. C. Lemmon ${ }^{85}$, V. Lenti ${ }^{105}$, E. Leogrande ${ }^{59}$, I. León Monzón ${ }^{121}$, H. León Vargas ${ }^{66}$, M. Leoncino ${ }^{26}$, P. Lévai ${ }^{138}$, S. Li ${ }^{7,72}$, X. Li ${ }^{14}$, J. Lien ${ }^{42}$, R. Lietava ${ }^{103}$, S. Lindal ${ }^{22}$, V. Lindenstruth ${ }^{43}$, C. Lippmann ${ }^{99}$, M. A. Lisa ${ }^{20}$, H. M. Ljunggren ${ }^{34}$,

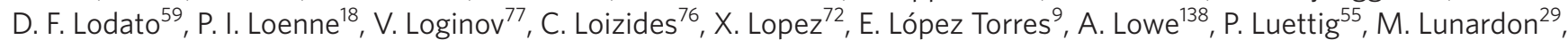
G. Luparello ${ }^{25}$, M. Lupi ${ }^{36}$, T. H. Lutz ${ }^{139}$, A. Maevskaya ${ }^{58}$, M. Mager ${ }^{36}$, S. Mahajann ${ }^{93}$, S. M. Mahmood ${ }^{22}$, A. Maire ${ }^{57}$, R. D. Majka ${ }^{139}$, M. Malaev ${ }^{88}$, I. Maldonado Cervantes ${ }^{65}$, L. Malinina ${ }^{68}$, D. Mal'Kevich ${ }^{60}$, P. Malzacher ${ }^{99}$, A. Mamonov ${ }^{101}$, V. Manko ${ }^{82}$, F. Manso ${ }^{72}$, V. Manzari ${ }^{36,105}$, Y. Mao ${ }^{7}$, M. Marchisone ${ }^{67,128,26}$, J. Mareš ${ }^{62}$, G. V. Margagliotti ${ }^{25}$, A. Margotti ${ }^{106}$, J. Margutti ${ }^{59}$, A. Marín ${ }^{99}$, C. Markert ${ }^{120}$, M. Marquard ${ }^{55}$, N. A. Martin ${ }^{99}$, P. Martinengo $0^{36}$, M. I. Martínez ${ }^{2}$,

G. Martínez García ${ }^{115}$, M. Martinez Pedreira ${ }^{36}$, A. Mas ${ }^{122}$, S. Masciocchi ${ }^{99}$, M. Masera ${ }^{26}$, A. Masoni ${ }^{107}$, A. Mastroserio ${ }^{33}$, A. Matyja ${ }^{119}$, C. Mayer 119 , J. Mazerr27, M. Mazzilli33, M. A. Mazzoni ${ }^{110}$, D. Mcdonald ${ }^{124}$, F. Meddi23, Y. Melikyan ${ }^{77}$, A. Menchaca-Rocha ${ }^{66}$, E. Meninno ${ }^{30}$, J. Mercado Pérez ${ }^{96}$, M. Meres $^{39}$, S. Mhlanga ${ }^{92}$, Y. Miake ${ }^{130}$, M. M. Mieskolainen ${ }^{47}$, K. Mikhaylov 60,68, L. Milano ${ }^{76,36}$, J. Milosevic ${ }^{22}$, A. Mischke ${ }^{59}$, A. N. Mishra ${ }^{50}$, T. Mishra ${ }^{63}$, D. Miśkowiec ${ }^{99}, J^{2}$ Mitra ${ }^{135}$, C. M. Mitu ${ }^{64}$, N. Mohammadi59, B. Mohanty ${ }^{81}$, L. Molnar ${ }^{57}$, L. Montaño Zetina ${ }^{11}$, E. Montes ${ }^{10}$, D. A. Moreira De Godoy ${ }^{56}$, L. A. P. Moreno ${ }^{2}$, S. Moretto ${ }^{29}$, A. Morreale ${ }^{115}$, A. Morsch ${ }^{36}$, V. Muccifora ${ }^{74}$, E. Mudnic 118 , D. Mühlheim ${ }^{56}$, S. Muhuri 135 , M. Mukherjee ${ }^{135}$, J. D. Mulligan ${ }^{139}$, M. G. Munhoz ${ }^{122}$, K. Münning $^{46}$, R. H. Munzer $95,37,55$, H. Murakami ${ }^{129}$, S. Murray ${ }^{67}$, L. Musa ${ }^{36}$, J. Musinsky ${ }^{61}$, B. Naik ${ }^{49}$, R. Nair ${ }^{79}$, B. K. Nandi ${ }^{49}$, R. Nania ${ }^{106}$, E. Nappi ${ }^{105}$, M. U. Naru ${ }^{16}$, H. Natal da Luz ${ }^{122}$, C. Nattrass ${ }^{127}$, S. R. Navarro ${ }^{2}$, K. Nayak ${ }^{81}$, R. Nayak ${ }^{49}$, T. K. Nayak ${ }^{135}$, S. Nazarenko ${ }^{101}$, A. Nedosekin ${ }^{60}$,

R. A. Negrao De Oliveira ${ }^{36}$, L. Nellen ${ }^{65}$, F. Ng ${ }^{124}$, M. Nicassio ${ }^{99}$, M. Niculescu ${ }^{64}$, J. Niedziela ${ }^{36}$, B. S. Nielsen ${ }^{83}$, S. Nikolaev $^{82}$, S. Nikulin ${ }^{82}$, V. Nikulin ${ }^{88}$, F. Noferini ${ }^{106,12}$, P. Nomokonov ${ }^{68}$, G. Nooren ${ }^{59}$, J. C. C. Noris ${ }^{2}$, J. Norman ${ }^{126}$, A. Nyanin ${ }^{82}$,

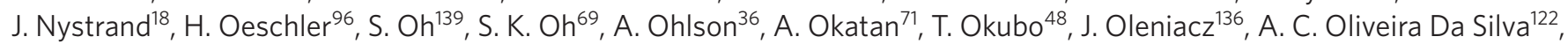
M. H. Oliver ${ }^{139}$, J. Onderwaater ${ }^{99}$, C. Oppedisano ${ }^{112}$, R. Orava ${ }^{47}$, M. Oravec ${ }^{117}$, A. Ortiz Velasquez ${ }^{65}$, A. Oskarsson ${ }^{34}$, J. Otwinowski1 ${ }^{119}$, K. Oyama ${ }^{96,78}$, M. Ozdemir ${ }^{55}$, Y. Pachmayer ${ }^{96}$, D. Pagano ${ }^{133}$, P. Pagano ${ }^{30}$, G. Paić ${ }^{65}$, S. K. Pal Pa $^{135}$, P. Palni ${ }^{7}$, J. Pan ${ }^{137}$, A. K. Pandey ${ }^{49}$, V. Papikyan', G. S. Pappalardo ${ }^{108}$, P. Pareek ${ }^{50}$, W. J. Park ${ }^{99}$, S. Parmar ${ }^{90}$, A. Passfeld ${ }^{56}$, V. Paticchio ${ }^{105}$, R. N. Patra ${ }^{135}$, B. Paul ${ }^{112}$, H. Pei ${ }^{7}$, T. Peitzmann ${ }^{59}$, X. Peng7, H. Pereira Da Costa ${ }^{15}$, D. Peresunko 82,77 , E. Perez Lezama ${ }^{55}$, V. Peskov ${ }^{55}$, Y. Pestov ${ }^{5}$, V. Petráček ${ }^{40}$, V. Petrov ${ }^{113}$, M. Petrovici ${ }^{80}$, C. Petta ${ }^{28}$, S. Piano ${ }^{111}$, M. Pikna ${ }^{39}$, P. Pillot ${ }^{115}$,

L. O. D. L. Pimentel ${ }^{83}$, O. Pinazza 106,36 , L. Pinsky ${ }^{124}$, D. B. Piyarathna ${ }^{124}$, M. Płoskon ${ }^{76}$, M. Planinic A $^{131}$, J. Pluta ${ }^{136}$, S. Pochybova ${ }^{138}$, P. L. M. Podesta-Lerma ${ }^{121}$, M. G. Poghosyan ${ }^{87}$, B. Polichtchouk ${ }^{113}$, N. Poljak ${ }^{131}$, W. Poonsawat ${ }^{116}$, A. Pop ${ }^{80}$, H. Poppenborg ${ }^{56}$, S. Porteboeuf-Houssais ${ }^{72}$, J. Porter ${ }^{76}$, J. Pospisil ${ }^{86}$, S. K. Prasad ${ }^{4}$, R. Preghenella106,36, F. Prino ${ }^{112}$, C. A. Pruneau ${ }^{137}$, I. Pshenichnov ${ }^{58}$, M. Puccio ${ }^{26}$, G. Puddu ${ }^{24}$, P. Pujahari ${ }^{137}$, V. Punin ${ }^{101}$, J. Putschke ${ }^{137}$, H. Qvigstad ${ }^{22}$, A. Rachevski111, S. Raha4, S. Rajput ${ }^{93}$, J. Rak' ${ }^{125}$, A. Rakotozafindrabe ${ }^{15}$, L. Ramello ${ }^{31}$, F. Rami ${ }^{57}$, R. Raniwala94, S. Raniwala94, S. S. Räsänen ${ }^{47}$, B. T. Rascanu ${ }^{55}$, D. Rathee ${ }^{90}$, I. Ravasenga ${ }^{26}$, K. F. Read ${ }^{127,87}$, K. Redlich ${ }^{79}$, R. J. Reed ${ }^{137}$, A. Rehman ${ }^{18}$, P. Reichelt ${ }^{55}$, F. Reidt ${ }^{36,96,}$ X. Ren7 , R. Renfordt ${ }^{55}$, A. R. Reolon ${ }^{74}$, A. Reshetin ${ }^{58}$, K. Reygers ${ }^{96}$, V. Riabov ${ }^{88}$, R. A. Ricci ${ }^{75}$, T. Richert $^{34}$, M. Richter ${ }^{22}$, P. Riedler ${ }^{36}$, W. Riegler ${ }^{36}$, F. Riggi ${ }^{28}$, C. Ristea ${ }^{64}$, M. Rodríguez Cahuantzi ${ }^{2}$, A. Rodriguez Manso ${ }^{84}$, K. Røed ${ }^{22}$, E. Rogochaya ${ }^{68}$, D. Rohr ${ }^{43}$, D. Röhrich ${ }^{18}$, F. Ronchetti ${ }^{36,74}$, L. Ronflette ${ }^{115}$, P. Rosnet ${ }^{72}$, A. Rossi ${ }^{29}$, F. Roukoutakis ${ }^{91}$, A. Roy ${ }^{50}$, C. Roy ${ }^{57}$, P. Roy ${ }^{102}$, A. J. Rubio Montero ${ }^{10}$, R. Ruii ${ }^{25}$, R. Russo ${ }^{26}$, E. Ryabinkin ${ }^{82}$, Y. Ryabov ${ }^{88}$, A. Rybicki ${ }^{119}$, S. Saarinen ${ }^{47}$, S. Sadhu ${ }^{135}$, S. Sadovsky ${ }^{113}$, K. Šafařík ${ }^{36}$, B. Sahlmuller ${ }^{55}$, P. Sahoo ${ }^{50}$, R. Sahoo ${ }^{50}$, S. Sahoo ${ }^{63}$, P. K. Sahu ${ }^{63}$, J. Saini ${ }^{135}$, S. Sakai ${ }^{74}$, M. A. Saleh ${ }^{137}$, J. Salzwedel ${ }^{20}$, S. Sambyal ${ }^{93}$, V. Samsonov 88,77 , L. Šándor ${ }^{61}$, A. Sandoval ${ }^{66}$, M. Sano ${ }^{130}$, D. Sarkar ${ }^{135}$, N. Sarkar ${ }^{135}$, P. Sarma ${ }^{45}$, E. Scapparone ${ }^{106,}$ F. Scarlassara ${ }^{29}$, C. Schiaua ${ }^{80}$, R. Schicker ${ }^{96}$, C. Schmidt ${ }^{99}$, H. R. Schmidt ${ }^{35}$, M. Schmidt ${ }^{35}$, S. Schuchmann ${ }^{55,96}$, J. Schukraft ${ }^{36}$, Y. Schutz ${ }^{36,115}$, K. Schwarz ${ }^{99}$, K. Schweda ${ }^{99}$, G. Scioli27, E. Scomparin ${ }^{112}$, R. Scott ${ }^{127}$, M. Šefčík ${ }^{41}$, J. E. Seger ${ }^{89}$, Y. Sekiguchi' ${ }^{129}$, D. Sekihata ${ }^{48}$, I. Selyuzhenkov ${ }^{99}$, K. Senosi ${ }^{67}$, S. Senyukov ${ }^{3,36}$, E. Serradilla ${ }^{10,66}$, A. Sevcenco ${ }^{64}$, A. Shabanov ${ }^{58}$, A. Shabetai ${ }^{115}$, O. Shadura ${ }^{3}$, R. Shahoyan ${ }^{36}$, A. Shangaraev ${ }^{113}$, A. Sharma ${ }^{93}$, M. Sharma ${ }^{93}$, M. Sharma ${ }^{93}$, N. Sharma ${ }^{127}$, A. I. Sheikh ${ }^{135}$, K. Shigaki ${ }^{48}$, Q. Shou ${ }^{7}$, K. Shtejer ${ }^{9,26}$, Y. Sibiriak ${ }^{82}$, S. Siddhanta ${ }^{107}$, K. M. Sielewicz ${ }^{36}$, T. Siemiarczuk ${ }^{79}$, D. Silvermyr ${ }^{34}$, C. Silvestre ${ }^{73}$, G. Simatovic ${ }^{131}$, G. Simonetti ${ }^{36}$, R. Singaraju ${ }^{135}$, R. Singh ${ }^{81}$, V. Singhal ${ }^{135}$, T. Sinha ${ }^{102}$, B. Sitar ${ }^{39}$, M. Sitta ${ }^{31}$, T. B. Skaali ${ }^{22}$, M. Slupecki ${ }^{125}$, N. Smirnov ${ }^{139}$, R. J. M. Snellings ${ }^{59}$, T. W. Snellmann ${ }^{125}$, J. Song ${ }^{98}$, M. Song ${ }^{140}$, Z. Song ${ }^{7}$, F. Soramel ${ }^{29}$, S. Sorensen ${ }^{127}$, F. Sozzi ${ }^{99}$, E. Spiriti ${ }^{74}$, I. Sputowska ${ }^{119}$, M. Spyropoulou-Stassinaki ${ }^{91}$, J. Stachel ${ }^{96}$, I. Stan ${ }^{64}$, P. Stankus ${ }^{87}$, E. Stenlund ${ }^{34}$, G. Steyn ${ }^{67}$, J. H. Stiller ${ }^{96}$, D. Stocco ${ }^{115}$, P. Strmen ${ }^{39}$, A. A. P. Suaide ${ }^{122}$, T. Sugitate ${ }^{48}$, C. Suire ${ }^{53}$, M. Suleymanov ${ }^{16}$, M. Suljic ${ }^{25 \dagger}$, R. Sultanov ${ }^{60}$, M. Šumbera ${ }^{86}$, S. Sumowidagdo 51 , S. Swain ${ }^{63}$, A. Szabo ${ }^{39}$, I. Szarka ${ }^{39}$, A. Szczepankiewicz ${ }^{136}$, M. Szymanski ${ }^{136}$, U. Tabassam ${ }^{16}$, J. Takahashi ${ }^{123}$, G. J. Tambave ${ }^{18}$, N. Tanaka ${ }^{130}$, M. Tarhini ${ }^{53}$, M. Tariq ${ }^{19}$, M. G. Tarzila ${ }^{80}$,

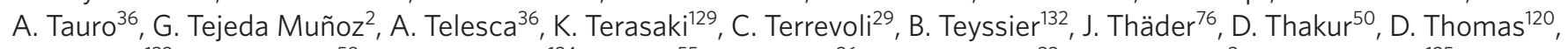
R. Tieulent ${ }^{132}$, A. Tikhonov ${ }^{58}$, A. R. Timmins ${ }^{124}$, A. Toia ${ }^{55}$, S. Trogolo ${ }^{26}$, G. Trombetta ${ }^{33}$, V. Trubnikov ${ }^{3}$, W. H. Trzaska ${ }^{125}$, T. Tsuji ${ }^{129}$, A. Tumkin ${ }^{101}$, R. Turrisi ${ }^{109}$, T. S. Tveter ${ }^{22}$, K. Ullaland ${ }^{18}$, A. Uras ${ }^{132}$, G. L. Usai ${ }^{24}$, A. Utrobicic ${ }^{131}$, M. Vala61, L. Valencia Palomo ${ }^{72}$, J. Van Der Maarel ${ }^{59}$, J. W. Van Hoorne ${ }^{36,114}$, M. van Leeuwen ${ }^{59}$, T. Vanat $^{86}$, P. Vande Vyvre ${ }^{36}$, D. Varga ${ }^{138,}$ A. Vargas ${ }^{2}$, M. Vargyas ${ }^{125}$, R. Varma ${ }^{49}$, M. Vasileiou ${ }^{91}$, A. Vasiliev ${ }^{82}$, A. Vauthier ${ }^{73}$, O. Vázquez Doce $^{95,37}$, V. Vechernin ${ }^{134}$, A. M. Veen ${ }^{59}$, A. Velure ${ }^{18}$, E. Vercellin ${ }^{26}$, S. Vergara Limón ${ }^{2}$, R. Vernet ${ }^{8}$, L. Vickovic $^{118}$, J. Viinikainen ${ }^{125}$, Z. Vilakazi ${ }^{128}$, 
O. Villalobos Baillie ${ }^{103}$, A. Villatoro Tello², A. Vinogradov ${ }^{82}$, L. Vinogradov ${ }^{134}$, T. Virgili30, V. Vislavicius ${ }^{34}$, Y. P. Viyogi ${ }^{135}$, A. Vodopyanov ${ }^{68}$, M. A. Völk1 $\left.\right|^{96}$, K. Voloshin ${ }^{60}$, S. A. Voloshin ${ }^{137}$, G. Volpe ${ }^{33,138}$, B. von Haller ${ }^{36}$, I. Vorobyev ${ }^{95,37}$, D. Vranic ${ }^{99,36,}$ J. Vrláková47 , B. Vulpescu ${ }^{72}$, B. Wagner ${ }^{18}$, J. Wagner ${ }^{99}$, H. Wang ${ }^{59}$, M. Wang ${ }^{7}$, D. Watanabe ${ }^{130}$, Y. Watanabe ${ }^{129}$, M. Weber ${ }^{36,114}$, S. G. Weber ${ }^{99}$, D. F. Weiser ${ }^{96}$, J. P. Wessels ${ }^{56}$, U. Westerhoff ${ }^{56}$, A. M. Whitehead ${ }^{92}$, J. Wiechula ${ }^{35}$, J. Wikne ${ }^{22}$, G. Wilk ${ }^{79}$, J. Wilkinson ${ }^{96}$, G. A. Willems ${ }^{56}$, M. C. S. Williams ${ }^{106}$, B. Windelband ${ }^{96}$, M. Winn ${ }^{96}$, S. Yalcin ${ }^{71}$, P. Yang ${ }^{7}$, S. Yano ${ }^{48}$, Z. Yin ${ }^{7}$, H. Yokoyama ${ }^{130}$, I.-K. Yoo ${ }^{98}$, J. H. Yoon ${ }^{52}$, V. Yurchenko ${ }^{3}$, A. Zaborowska ${ }^{136}$, V. Zaccolo ${ }^{83}$, A. Zamann' ${ }^{16}$ C. Zampolli ${ }^{106,36,}$ H. J. C. Zanoli ${ }^{122}$, S. Zaporozhets ${ }^{68}$, N. Zardoshti ${ }^{103}$, A. Zarochentsev ${ }^{134}$, P. Závada ${ }^{62}$, N. Zaviyalov ${ }^{101}$, H. Zbroszczyk ${ }^{136}$, I. S. Zgura ${ }^{64}$, M. Zhalov ${ }^{88}$, H. Zhang ${ }^{18,7}$, X. Zhang ${ }^{76,7}$, Y. Zhang ${ }^{7}$, C. Zhang ${ }^{59}$, Z. Zhang ${ }^{7}$, C. Zhao ${ }^{22}$, N. Zhigareva ${ }^{60}$, D. Zhou' Y. Zhou ${ }^{83}$, Z. Zhou ${ }^{18}$, H. Zhu' ${ }^{18,7}$, J. Zhu ${ }^{7,115}$, A. Zichichi' ${ }^{27,12}$, A. Zimmermann ${ }^{96}$, M. B. Zimmermann ${ }^{56,36}$, G. Zinovjev ${ }^{3}$, M. Zyzak $^{43}$

${ }^{1}$ A.I. Alikhanyan National Science Laboratory (Yerevan Physics Institute) Foundation, Yerevan, Armenia. ${ }^{2}$ Benemérita Universidad Autónoma de Puebla, Puebla, Mexico. ${ }^{3}$ Bogolyubov Institute for Theoretical Physics, Kiev, Ukraine. ${ }^{4}$ Bose Institute, Department of Physics and Centre for Astroparticle Physics and Space Science (CAPSS), Kolkata, India. ${ }^{5}$ Budker Institute for Nuclear Physics, Novosibirsk, Russia. ${ }^{6}$ California Polytechnic State University, San Luis Obispo California, USA. ${ }^{7}$ Central China Normal University, Wuhan, China. ${ }^{8}$ Centre de Calcul de I'IN2P3, Villeurbanne, France. ${ }^{9}$ Centro de Aplicaciones Tecnológicas y Desarrollo Nuclear (CEADEN), Havana, Cuba. ${ }^{10}$ Centro de Investigaciones Energéticas Medioambientales y Tecnológicas (CIEMAT), Madrid, Spain. ${ }^{11}$ Centro de Investigación y de Estudios Avanzados (CINVESTAV), Mexico City and Mérida, Mexico. ${ }^{12}$ Centro Fermi - Museo Storico della Fisica e Centro Studi e Ricerche "Enrico Fermi", Rome, Italy. ${ }^{13}$ Chicago State University, Chicago, Illinois, USA. ${ }^{14}$ China Institute of Atomic Energy, Beijing, China. ${ }^{15}$ Commissariat à I'Energie Atomique, IRFU, Saclay, France. ${ }^{16}$ COMSATS Institute of Information Technology (CIIT), Islamabad, Pakistan. ${ }^{17}$ Departamento de Física de Partículas and IGFAE, Universidad de Santiago de Compostela, Santiago de Compostela, Spain. ${ }^{18}$ Department of Physics and Technology, University of Bergen, Bergen, Norway. ${ }^{19}$ Department of Physics, Aligarh Muslim University, Aligarh, India. ${ }^{20}$ Department of Physics, Ohio State University, Columbus, Ohio, USA. ${ }^{21}$ Department of Physics, Sejong University, Seoul, South Korea. ${ }^{22}$ Department of Physics, University of Oslo, Oslo, Norway. ${ }^{23}$ Dipartimento di Fisica dell'Università 'La Sapienza' and Sezione INFN Rome, Italy. ${ }^{24}$ Dipartimento di Fisica dell'Università and Sezione INFN, Cagliari, Italy. ${ }^{25}$ Dipartimento di Fisica dell'Università and Sezione INFN, Trieste, Italy. ${ }^{26}$ Dipartimento di Fisica dell'Università and Sezione INFN, Turin, Italy. ${ }^{27}$ Dipartimento di Fisica e Astronomia dell'Università and Sezione INFN, Bologna, Italy. ${ }^{28}$ Dipartimento di Fisica e Astronomia dell'Università and Sezione INFN, Catania, Italy. ${ }^{29}$ Dipartimento di Fisica e Astronomia dell'Università and Sezione INFN, Padova, Italy. ${ }^{30}$ Dipartimento di Fisica 'E.R. Caianiello' dell'Università and Gruppo Collegato INFN, Salerno, Italy. ${ }^{31}$ Dipartimento di Scienze e Innovazione Tecnologica dell'Università del Piemonte Orientale and Gruppo Collegato INFN, Alessandria, Italy. ${ }^{32}$ Dipartimento DISAT del Politecnico and Sezione INFN, Turin, Italy. ${ }^{33}$ Dipartimento Interateneo di Fisica 'M. Merlin' and Sezione INFN, Bari, Italy. ${ }^{34}$ Division of Experimental High Energy Physics, University of Lund, Lund, Sweden. ${ }^{35}$ Eberhard Karls Universität Tübingen, Tübingen, Germany. ${ }^{36}$ European Organization for Nuclear Research (CERN), Geneva, Switzerland. ${ }^{37}$ Excellence Cluster Universe, Technische Universität München, Munich, Germany. ${ }^{38}$ Faculty of Engineering, Bergen University College, Bergen, Norway. ${ }^{39}$ Faculty of Mathematics, Physics and Informatics, Comenius University, Bratislava, Slovakia. ${ }^{40}$ Faculty of Nuclear Sciences and Physical Engineering, Czech Technical University in Prague, Prague, Czech Republic. ${ }^{41}$ Faculty of Science, P.J. Šafárik University, Košice, Slovakia. ${ }^{42}$ Faculty of Technology, Buskerud and Vestfold University College, Vestfold, Norway. ${ }^{43}$ Frankfurt Institute for Advanced Studies, Johann Wolfgang Goethe-Universität Frankfurt, Frankfurt, Germany.

${ }^{44}$ Gangneung-Wonju National University, Gangneung, South Korea. ${ }^{45}$ Gauhati University, Department of Physics, Guwahati, India. ${ }^{46}$ Helmholtz-Institut für Strahlen- und Kernphysik, Rheinische Friedrich-Wilhelms-Universität Bonn, Bonn, Germany. ${ }^{47}$ Helsinki Institute of Physics (HIP), Helsinki, Finland. ${ }^{48}$ Hiroshima University, Hiroshima, Japan. ${ }^{49}$ Indian Institute of Technology Bombay (IIT), Mumbai, India. ${ }^{50}$ Indian Institute of Technology Indore, Indore (IITI), India. ${ }^{51}$ Indonesian Institute of Sciences, Jakarta, Indonesia. ${ }^{52}$ Inha University, Incheon, South Korea. ${ }^{53}$ Institut de Physique Nucléaire d'Orsay (IPNO), Université Paris-Sud, CNRS-IN2P3, Orsay, France. ${ }^{54}$ Institut für Informatik, Johann Wolfgang Goethe-Universität Frankfurt, Frankfurt, Germany. ${ }^{55}$ Institut für Kernphysik, Johann Wolfgang Goethe-Universität Frankfurt, Frankfurt, Germany. ${ }^{56}$ Institut für Kernphysik, Westfälische Wilhelms-Universität Münster, Münster, Germany. ${ }^{57}$ Institut Pluridisciplinaire Hubert Curien (IPHC), Université de Strasbourg, CNRS-IN2P3, Strasbourg, France. ${ }^{58}$ Institute for Nuclear Research, Academy of Sciences, Moscow, Russia. ${ }^{59}$ Institute for Subatomic Physics of Utrecht University, Utrecht, Netherlands. ${ }^{60}$ Institute for Theoretical and Experimental Physics, Moscow, Russia. ${ }^{61}$ Institute of Experimental Physics, Slovak Academy of Sciences, Košice, Slovakia. ${ }^{62}$ Institute of Physics, Academy of Sciences of the Czech Republic, Prague, Czech Republic. ${ }^{63}$ Institute of Physics, Bhubaneswar, India. ${ }^{64}$ Institute of Space Science (ISS), Bucharest, Romania. ${ }^{65}$ Instituto de Ciencias Nucleares, Universidad Nacional Autónoma de México, Mexico City, Mexico. ${ }^{66}$ Instituto de Física, Universidad Nacional Autónoma de México, Mexico City, Mexico. ${ }^{67}$ iThemba LABS, National Research Foundation, Somerset West, South Africa. ${ }^{68}$ Joint Institute for Nuclear Research (JINR), Dubna, Russia. ${ }^{69}$ Konkuk University, Seoul, South Korea. ${ }^{70}$ Korea Institute of Science and Technology Information, Daejeon, South Korea. ${ }^{71}$ KTO Karatay University, Konya, Turkey. ${ }^{72}$ Laboratoire de Physique Corpusculaire (LPC), Clermont Université, Université Blaise Pascal, CNRS-IN2P3, Clermont-Ferrand, France. ${ }^{73}$ Laboratoire de Physique Subatomique et de Cosmologie, Université Grenoble-Alpes, CNRS-IN2P3, Grenoble, France. ${ }^{74}$ Laboratori Nazionali di Frascati, INFN, Frascati, Italy. ${ }^{75}$ Laboratori Nazionali di Legnaro, INFN, Legnaro, Italy. ${ }^{76}$ Lawrence Berkeley National Laboratory, Berkeley, California, USA. ${ }^{77}$ Moscow Engineering Physics Institute, Moscow, Russia. ${ }^{78}$ Nagasaki Institute of Applied Science, Nagasaki, Japan. ${ }^{79}$ National Centre for Nuclear Studies, Warsaw, Poland. ${ }^{80}$ National Institute for Physics and Nuclear Engineering, Bucharest, Romania. ${ }^{81}$ National Institute of Science Education and Research, Bhubaneswar, India. ${ }^{82}$ National Research Centre Kurchatov Institute, Moscow, Russia. ${ }^{83}$ Niels Bohr Institute, University of Copenhagen, Copenhagen, Denmark. ${ }^{84}$ Nikhef, Nationaal instituut voor subatomaire fysica, Amsterdam, Netherlands. ${ }^{85}$ Nuclear Physics Group, STFC Daresbury Laboratory, Daresbury, UK. ${ }^{86}$ Nuclear Physics Institute, Academy of Sciences of the Czech Republic, Řež u Prahy, Czech Republic. ${ }^{87}$ Oak Ridge National Laboratory, Oak Ridge, Tennessee, USA. ${ }^{88}$ Petersburg Nuclear Physics Institute, Gatchina, Russia. ${ }^{89}$ Physics Department, Creighton University, Omaha, Nebraska, USA. ${ }^{90}$ Physics Department, Panjab University, Chandigarh, India. ${ }^{91}$ Physics Department, University of Athens, Athens, Greece. ${ }^{92}$ Physics Department, University of Cape Town, Cape Town, South Africa. ${ }^{93}$ Physics Department, University of Jammu, Jammu, India. ${ }^{94}$ Physics Department, University of Rajasthan, Jaipur, India. ${ }^{95}$ Physik Department, Technische Universität München, Munich, Germany. ${ }^{96}$ Physikalisches Institut, Ruprecht-Karls-Universität Heidelberg, Heidelberg, Germany. ${ }^{97}$ Purdue University, West Lafayette, Indiana, USA. ${ }^{98}$ Pusan National University, Pusan, South Korea. ${ }^{99}$ Research Division and ExtreMe Matter Institute EMMI, GSI Helmholtzzentrum für Schwerionenforschung, Darmstadt, Germany. ${ }^{100}$ Rudjer Bošković Institute, Zagreb, Croatia. ${ }^{101}$ Russian Federal Nuclear Center (VNIIEF), Sarov, Russia. ${ }^{102}$ Saha Institute of Nuclear Physics, Kolkata, India. ${ }^{103}$ School of Physics and Astronomy, University of Birmingham, Birmingham, UK. ${ }^{104}$ Sección Física, Departamento de Ciencias, Pontificia Universidad Católica del Perú, Lima, Peru. ${ }^{105}$ Sezione INFN, Bari, Italy. ${ }^{106}$ Sezione INFN, Bologna, Italy. ${ }^{107}$ Sezione INFN, Cagliari, Italy. ${ }^{108}$ Sezione INFN, Catania, Italy. ${ }^{109}$ Sezione INFN, Padova, Italy. ${ }^{110}$ Sezione INFN, Rome, Italy. ${ }^{111}$ Sezione INFN, Trieste, Italy. ${ }^{112}$ Sezione INFN, Turin, Italy. ${ }^{113}$ SSC IHEP of NRC Kurchatov institute, Protvino, Russia. ${ }^{114}$ Stefan Meyer Institut für Subatomare Physik (SMI), Vienna, Austria. ${ }^{115}$ SUBATECH, Ecole des Mines de Nantes, Université de Nantes, CNRS-IN2P3, Nantes, France. ${ }^{116}$ Suranaree University of Technology, Nakhon Ratchasima, Thailand. ${ }^{117}$ Technical University of Košice, Košice, Slovakia. ${ }^{118}$ Technical University of Split FESB, Split, Croatia. ${ }^{119}$ The Henryk Niewodniczanski Institute of Nuclear Physics, Polish Academy of Sciences, Cracow, Poland. ${ }^{120}$ The University of Texas at Austin, Physics Department, Austin, Texas, USA. ${ }^{121}$ Universidad Autónoma de Sinaloa, Culiacán, Mexico. 
${ }^{122}$ Universidade de São Paulo (USP), São Paulo, Brazil. ${ }^{123}$ Universidade Estadual de Campinas (UNICAMP), Campinas, Brazil. ${ }^{124}$ University of Houston, Houston, Texas, USA. ${ }^{125}$ University of Jyväskylä, Jyväskylä, Finland. ${ }^{126}$ University of Liverpool, Liverpool, UK. ${ }^{127}$ University of Tennessee, Knoxville, Tennessee, USA. ${ }^{128}$ University of the Witwatersrand, Johannesburg, South Africa. ${ }^{129}$ University of Tokyo, Tokyo, Japan. ${ }^{130}$ University of Tsukuba, Tsukuba, Japan. ${ }^{131}$ University of Zagreb, Zagreb, Croatia. ${ }^{132}$ Université de Lyon, Université Lyon 1, CNRS/IN2P3, IPN-Lyon, Villeurbanne, France. ${ }^{133}$ Università di Brescia, Brescia, Italy. ${ }^{134}$ V. Fock Institute for Physics, St. Petersburg State University, St. Petersburg, Russia. ${ }^{135}$ Variable Energy Cyclotron Centre, Kolkata, India. ${ }^{136}$ Warsaw University of Technology, Warsaw, Poland. ${ }^{137}$ Wayne State University, Detroit, Michigan, USA. ${ }^{138}$ Wigner Research Centre for Physics, Hungarian Academy of Sciences, Budapest, Hungary. ${ }^{139}$ Yale University, New Haven, Connecticut, USA. ${ }^{140}$ Yonsei University, Seoul, South Korea.

${ }^{141}$ Zentrum für Technologietransfer und Telekommunikation (ZTT), Fachhochschule Worms, Worms, Germany. "Deceased. "Present addresses: Georgia State University, Atlanta, Georgia, USA (M.E.C.); Department of Applied Physics, Aligarh Muslim University, Aligarh, India (M.M.K.); M.V. Lomonosov Moscow State University, D.V. Skobeltsyn Institute of Nuclear, Physics, Moscow, Russia (L.M.). ${ }^{\star}$--mail: alice-publications@cern.ch 
Table 1 | Event multiplicity classes, their corresponding fraction of the INEL $>0$ cross-section $\left(\sigma / \sigma_{\text {INEL }}>0\right)$ and their corresponding $\left\langle\mathrm{d} N_{\mathrm{ch}} / \mathrm{d} \eta\right\rangle$ at midrapidity $(|\eta|<0.5)$.

\begin{tabular}{lllllllllll} 
Class name & $\mathbf{I}$ & II & III & IV & V & VI & VII & VIII & IX & X \\
\hline$\sigma / \sigma_{\text {INEL }>0}$ & $0-0.95 \%$ & $0.95-4.7 \%$ & $4.7-9.5 \%$ & $9.5-14 \%$ & $14-19 \%$ & $19-28 \%$ & $28-38 \%$ & $38-48 \%$ & $48-68 \%$ & $68-100 \%$ \\
$\left\langle\mathrm{~d} N_{\mathrm{ch}} / \mathrm{d} \eta\right\rangle$ & $21.3 \pm 0.6$ & $16.5 \pm 0.5$ & $13.5 \pm 0.4$ & $11.5 \pm 0.3$ & $10.1 \pm 0.3$ & $8.45 \pm 0.25$ & $6.72 \pm 0.21$ & $5.40 \pm 0.17$ & $3.90 \pm 0.14$ & $2.26 \pm 0.12$ \\
\hline
\end{tabular}

The value of $\left\langle\mathrm{d} N_{\mathrm{ch}} / \mathrm{d} \eta\right\rangle$ in the inclusive (INEL $>0$ ) class is $5.96 \pm 0.23$. The uncertainties are the quadratic sum of statistical and systematic contributions and represent standard deviations.

Table 2 | Main sources and values of the relative systematic uncertainties (standard deviations expressed in \%) of the $p_{\mathrm{T}}$-differential yields.

\begin{tabular}{|c|c|c|c|c|c|c|c|c|c|c|c|c|}
\hline \multirow{2}{*}{$\begin{array}{l}\text { Hadron } \\
p_{\mathrm{T}}(\mathrm{GeV} / \mathrm{c})\end{array}$} & \multicolumn{3}{|c|}{$K_{\mathrm{S}}^{0}$} & \multicolumn{3}{|c|}{$\Lambda(\bar{\Lambda})$} & \multicolumn{3}{|c|}{$\Xi^{-}\left(\bar{\Xi}^{+}\right)$} & \multicolumn{3}{|c|}{$\Omega^{-}\left(\bar{\Omega}^{+}\right)$} \\
\hline & 0.05 & 6.2 & 11.0 & $\overline{0.5}$ & 3.7 & 7.2 & $\overline{0.8}$ & 2.1 & 5.8 & 1.2 & 2.8 & 4.7 \\
\hline Material budget & 4.0 & 4.0 & 4.0 & 4.0 & 4.0 & 4.0 & 4.0 & 4.0 & 4.0 & 4.0 & 4.0 & 4.0 \\
\hline Transport code & \multicolumn{3}{|c|}{ Negligible } & 1.0 & 1.0 & 1.0 & 1.0 & 1.0 & 1.0 & 1.0 & 1.0 & 1.0 \\
\hline Track selection & 1.0 & 5.0 & 0.8 & 0.2 & 5.9 & 4.3 & 0.4 & 0.3 & 2.2 & 0.8 & 0.6 & 4.1 \\
\hline Topological selection & 2.6 & 1.1 & 2.3 & 0.8 & 0.6 & 3.2 & 3.1 & 2.0 & 4.0 & 5.0 & 5.6 & 8.1 \\
\hline Particle identification & 0.1 & 0.1 & 0.1 & 0.2 & 0.2 & 3.0 & 1.0 & 0.2 & 1.2 & 1.1 & 1.7 & 3.2 \\
\hline Efficiency determination & 2.0 & 2.0 & 2.0 & 2.0 & 2.0 & 2.0 & 2.0 & 2.0 & 2.0 & 2.0 & 2.0 & 2.0 \\
\hline Signal extraction & 1.5 & 1.2 & 3.6 & 0.6 & 0.7 & 3.0 & 1.5 & 0.2 & 1.0 & 3.2 & 2.5 & 2.3 \\
\hline Proper lifetime & 1.3 & 0.1 & 0.2 & 0.3 & 2.3 & 0.1 & 0.9 & 0.1 & 0.1 & 2.2 & 0.7 & 0.7 \\
\hline Competing decay rejection & Negl. & 0.7 & 1.3 & Negl. & 1.0 & 6.2 & \multirow{2}{*}{\multicolumn{3}{|c|}{$\begin{array}{l}\text { Not applicable } \\
\text { Negligible }\end{array}$}} & 0.2 & 4.2 & 5.2 \\
\hline Feed-down correction & \multicolumn{3}{|c|}{ Not applicable } & 3.3 & 2.1 & 4.3 & & & & & gligit & \\
\hline Total & 5.6 & 6.9 & 6.4 & 5.8 & 8.2 & 11.2 & 5.9 & 5.0 & 6.7 & 7.9 & 9.0 & 12.1 \\
\hline Common ( $N_{\text {ch }}$-independent $)$ & 5.0 & 5.9 & 4.4 & 5.4 & 7.8 & 9.9 & 5.2 & 4.5 & 6.2 & 7.3 & 8.7 & 11.6 \\
\hline
\end{tabular}

The values are reported for low, intermediate and high $p_{\mathrm{T}}$. The sums of the contributions common to all event classes are listed separately as $N_{\mathrm{ch}}$-independent systematics.

\section{Methods}

A detailed description of the ALICE detector and of its performance can be found in refs 26,33 . We briefly outline the main detectors utilized for this analysis. The V0 detectors are two scintillator hodoscopes employed for triggering, background suppression and event-class determination. They are placed on either side of the interaction region at $z=3.3 \mathrm{~m}$ and $z=-0.9 \mathrm{~m}$, covering the pseudorapidity regions $2.8<\eta<5.1$ and $-3.7<\eta<-1.7$, respectively. Vertex reconstruction, central-barrel tracking and charged-hadron identification are performed with the Inner Tracking System (ITS) and the Time-Projection Chamber (TPC), which are located inside a solenoidal magnet providing a $0.5 \mathrm{~T}$ magnetic field. The ITS is composed of six cylindrical layers of high-resolution silicon tracking detectors. The innermost layers consist of two arrays of hybrid silicon pixel detectors (SPD) located at average radii 3.9 and $7.6 \mathrm{~cm}$ from the beam axis and covering $|\eta|<2.0$ and $|\eta|<1.4$, respectively. The TPC is a large cylindrical drift detector of radial and longitudinal size of about $85<r<250 \mathrm{~cm}$ and $-250<z<250 \mathrm{~cm}$, respectively. It provides charged-hadron identification information via ionization energy loss in the fill gas.

The data were collected in 2010 using a minimum-bias trigger requiring a hit in either the V0 scintillators or in the SPD detector, in coincidence with the arrival of proton bunches from both directions. The contamination from beam-induced background is removed offline by using the timing information and correlations in the V0 and SPD detectors, as discussed in detail in ref. 33. Events used for the data analysis are further required to have a reconstructed vertex within $|z|<10 \mathrm{~cm}$. Events containing more than one distinct vertex are tagged as pileup and are discarded. The remaining pileup fraction is estimated to be negligible, ranging from about $10^{-4}$ to $10^{-2}$ for the lowest and highest multiplicity classes, respectively. A total of about 100 million events has been utilized for the analysis.

The mean pseudorapidity densities of primary charged particles $\left\langle\mathrm{d} N_{\mathrm{ch}} / \mathrm{d} \eta\right\rangle$ are measured at midrapidity, $|\eta|<0.5$, for each event class using the technique described in ref. 34. The $\left\langle\mathrm{d} N_{\mathrm{ch}} / \mathrm{d} \eta\right\rangle$ values, corrected for acceptance and efficiency, as well as for contamination from secondary particles and combinatorial background, are listed in Table 1. The relative RMS width of the corresponding multiplicity distributions ranges from $68 \%$ to $30 \%$ for the lowest and highest multiplicity classes, respectively. The corresponding fractions of the INEL $>0$ cross-section are also summarized inTable 1.

Strange $K_{\mathrm{s}}^{0}, \Lambda$ and $\bar{\Lambda}$ and multi-strange $\Xi^{-}, \bar{\Xi}^{+}, \Omega^{-}$and $\bar{\Omega}^{+}$candidates are reconstructed via topological selection criteria and invariant-mass analysis of their characteristic weak decays ${ }^{35}$ (BR is branching ratio):

$$
\begin{gathered}
K_{\mathrm{S}}^{0} \rightarrow \pi^{+}+\pi^{-} \quad \mathrm{BR}=(69.20 \pm 0.05) \% \\
\Lambda(\bar{\Lambda}) \rightarrow p(\bar{p})+\pi^{-}\left(\pi^{+}\right) \quad \mathrm{BR}=(63.9 \pm 0.5) \%
\end{gathered}
$$

$$
\Xi^{-}\left(\bar{\Xi}^{+}\right) \rightarrow \Lambda(\bar{\Lambda})+\pi^{-}\left(\pi^{+}\right) \quad \mathrm{BR}=(99.887 \pm 0.035) \%
$$

$$
\Omega^{-}\left(\bar{\Omega}^{+}\right) \rightarrow \Lambda(\bar{\Lambda})+\mathrm{K}^{-}\left(\mathrm{K}^{+}\right) \quad \mathrm{BR}=(67.8 \pm 0.7) \%
$$

Details on the analysis technique are described in refs 10,36,37. The results are corrected for detector acceptance and reconstruction efficiency calculated using events from the PYTHIA6 (tune Perugia 0) MC generator ${ }^{38}$ with particle transport performed via a GEANT3 (ref. 39) simulation of the ALICE detector. The contamination to $\Lambda(\bar{\Lambda})$ yields from weak decays of charged and neutral $\Xi$ baryons (feed-down) is subtracted using a data-driven approach ${ }^{10}$. The study of systematic uncertainties follows the analysis described in refs 10,36,37.

Contributions common to all event classes ( $N_{\mathrm{ch}}$-independent) are estimated and removed to determine the remaining uncertainties which are uncorrelated across different multiplicity intervals. The main sources of systematic uncertainty and their corresponding values are summarized in Table 2 . The results on pion and proton production have been obtained following the analysis method discussed in ref. 40 .

Data availability. All data shown in histograms and plots are publicly available from HEPdata (https://hepdata.net).

\section{References}

33. Abelev, B. et al. (ALICE Collaboration) Performance of the ALICE experiment at the CERN LHC. Int. J. Mod. Phys. A 29, 1430044 (2014).

34. Abelev, B. et al. (ALICE Collaboration) Pseudorapidity density of charged particles in $\mathrm{p}+\mathrm{Pb}$ collisions at $\sqrt{s_{\mathrm{NN}}}=5.02 \mathrm{TeV}$. Phys. Rev. Lett. 110, 032301 (2013)

35. Olive, K. A. et al. (Particle Data Group Collaboration) Review of particle physics. Chin. Phys. C 38, 090001 (2014).

36. Aamodt, K. et al. (ALICE Collaboration) Strange particle production in proton-proton collisions at $\sqrt{s}=0.9 \mathrm{TeV}$ with ALICE at the LHC. Eur. Phys. J. C 71, 1594 (2011).

37. Abelev, B. et al. (ALICE Collaboration) Multi-strange baryon production in $\mathrm{pp}$ collisions at $\sqrt{s}=7 \mathrm{TeV}$ with ALICE. Phys. Lett. B 712, 309-318 (2012).

38. Skands, P. Z. Tuning Monte Carlo generators: the Perugia tunes. Phys. Rev. D 82, 074018 (2010)

39. Brun, R. et al. GEANT Detector Description and Simulation Tool (CERN, 1993); http://cds.cern.ch/record/1082634

40. Adam, J. et al. (ALICE Collaboration) Measurement of pion, kaon and proton production in proton-proton collisions at $\sqrt{s}=7 \mathrm{TeV}$. Eur. Phys. J. C 75, 226 (2015). 